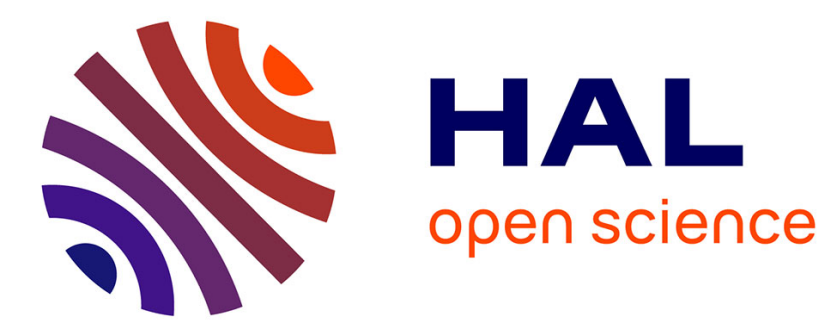

\title{
Multi-Dimensional Harmonic Balance With Uncertainties Applied to Rotor Dynamics
}

\author{
Jérôme Didier, Jean-Jacques Sinou, Béatrice Faverjon
}

\section{To cite this version:}

Jérôme Didier, Jean-Jacques Sinou, Béatrice Faverjon. Multi-Dimensional Harmonic Balance With Uncertainties Applied to Rotor Dynamics. Journal of Vibration and Acoustics, 2012, 134 (6), 10.1115/1.4006645 . hal-00732129

\section{HAL Id: hal-00732129 \\ https://hal.science/hal-00732129}

Submitted on 1 Oct 2018

HAL is a multi-disciplinary open access archive for the deposit and dissemination of scientific research documents, whether they are published or not. The documents may come from teaching and research institutions in France or abroad, or from public or private research centers.
L'archive ouverte pluridisciplinaire HAL, est destinée au dépôt et à la diffusion de documents scientifiques de niveau recherche, publiés ou non, émanant des établissements d'enseignement et de recherche français ou étrangers, des laboratoires publics ou privés. 


\section{Multi-Dimensional Harmonic Balance With Uncertainties Applied to Rotor Dynamics}

This paper describes the coupling of a Multi-Dimensional Harmonic Balance Method (MHBM) with a Polynomial Chaos Expansion (PCE) to determine the dynamic response of quasi-periodic dynamic systems subjected to multiple excitations and uncertainties. The proposed method will be applied to a rotor system excited at its support. Uncertainties considered include both material and geometrical parameters as well as excitation sources. To demonstrate the effectiveness and validity of the proposed numerical approach, the results that include mean, variation of the response, envelopes of the Frequency Response Functions and orbits will be systematically compared to a classical Monte Carlo approach.
J. Didier

e-mail: jerome.didier@ec-lyon.fr

J.-J. Sinou

e-mail: jean-jacques.sinou@ec-lyon.fr

LTDS

UMR-CNRS 5513

Ecole Centrale de Lyon,

69134, France

B. Faverjon

LaMCoS,

UMR-CNRS 5259

INSA Lyon,

69621, France

e-mail: beatrice.faverjon@insa-lyon.fr

\section{Introduction}

This study proposes to focus on quasi-periodic dynamic responses of rotating systems that can be subjected to various excitation sources such as unbalance, vibration of the system's support, or the presence of faults. Also, during the design stage of such mechanical systems, it is often necessary to consider uncertainties on physical parameters such as excitation sources acting on the system or geometric and material parameters. To be close to the reality, we propose to consider physical parameters as random and to develop a new approach to obtain the random quasiperiodic dynamics subject to multi-frequency excitations. The proposed numerical technique is based on the extension of a Multi-Dimensional Harmonic Balance Method (MHBM) with the Polynomial Chaos Expansion (PCE). The results can then estimate both the evolutions of mean and variation of the quasi-periodic response (or of each harmonic component), and the orbits obtained for a rotating speed. The methodology of the Polynomial Chaos Expansion with the Harmonic Balance Method (HBM) has been previously proposed for the quantification of uncertainty effects on the variability of the response in rotor systems with multi-faults [1] (such as unbalance, asymmetric shaft, bow, parallel, and angular misalignments). So, the present study is an extension of this methodology to obtain the quasi-periodic dynamic response of a rotor system subjected to multi-frequency excitations in the presence of uncertainty on several physical parameters.

Thus, the proposed methodology is to express the response in the form of multiple Fourier series [2] associated with a twodimensional time domain. As part of the study, it allows the inclusion of both multiple frequency excitations and contributions of faults. Concerning more specifically taking into account uncertainties in this type of problem, they can be made using the classical perturbation methods, or the Monte Carlo method, or the Polynomial Chaos Expansion. The perturbation methods are based on the development of random quantities in Taylor or Neumann series $[3,4]$. These methods give good results for small variations but seem not suitable to solve a problem of dynamics for frequencies near resonance. The Monte Carlo method can take into account the uncertainties in a deterministic model by generating samples. This method is suitable for dealing with linear and nonlinear problems; however, it is costly in computation time due to the need of one large set of samples. Then, it seems more appropriate to propose the use of the Polynomial Chaos Expansion [5], based on the representation of random processes and variables on a basis of orthogonal polynomials called Polynomial Chaoses. In this study, the uncertainties apply on the linear and parametric terms of stiffness, damping, gyroscopic, and mass contributions. To illustrate the effectiveness of the proposed methodology, the results are compared with those obtained by using the Monte Carlo simulations (MCS). All the tests are made for a rotor system subjected to multiple excitations.

The presence of uncertainties on the excitation forces and on the materials and geometric characteristics of the system are examined in details. Variations of physical parameters considered in this paper relate to the magnitude of the force generated by unbalance, to excitation at the base of the system [6] for which the amplitude is considered random, and to the Young's modulus of the rotor shaft. Moreover, we consider the presence of asymmetry from the geometry of the rotor (i.e. asymmetry of the section of the shaft) generating a parametric stiffness contribution (See Ref. [7], pages 181-187). In this case, the variation will focus on the depth of the asymmetry.

The study is composed as follows: after a brief description of the rotor and the various faults considered, we present the MultiDimensional Harmonic Balance Method with the Polynomial Chaos Expansion to determine all the random quasi-periodic dynamic responses of the mechanical system subject to multifrequency excitations and uncertainties. The last section presents the various outcomes that allow for calculations of evolutions of the Frequency Response Functions according to the two excitation frequencies (i.e. unbalance and excitation of the rotor's base) and the harmonic components of the rotor response for a fixed frequency excitation of the rotor's base. We will show that it is possible to obtain the responses of the Frequency Response Functions for each multiple Fourier order with a two-dimensional time domain, and orbits for various rotational speeds. Systematic comparisons with the Monte Carlo simulations will be proposed to demonstrate the effectiveness and relevance of the proposed approach.

\section{Rotor Model}

2.1 Equation of the Rotor System. The rotor system studied is shown in Fig. 1. It consists of a shaft and two discs. The shaft is discretized into 10 beam finite elements (Euler formulation) with 


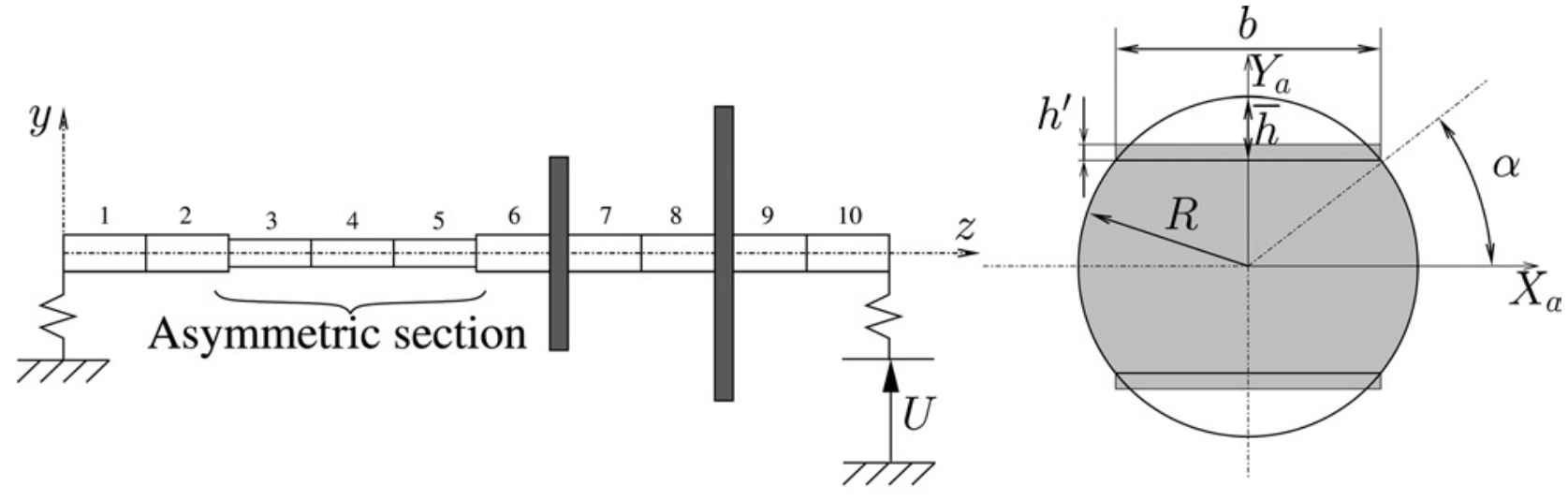

(a)

(b)

Fig. 1 Rotor system with the cross section of the asymmetric shaft (a) rotor with asymmetric shaft (b) uncertainty on the shaft section

4 degrees of freedom at each node. The degrees of freedom considered are denoted by $\left[\begin{array}{llll}v & w & \theta & \psi\end{array}\right]^{T}$ where $v$ and $w$ are associated with translations along $x$ and $y, \theta$ and $\psi$ associated with the following rotations $x$ and $y$ (see Fig. 2).

Moreover, the shaft has a section on asymmetrical elements numbered 3, 4, and 5 and a circular section on the other elements (see Fig. 1). The system is supported at its ends by two bearings modeled by linear stiffness. Finally, the base of the right bearing is subjected to a vibration amplitude $U_{0}$ and pulsation $\omega_{b}$. Specific modeling associated with the excitation of the rotor base will be detailed in the next section. After assembling the various elements of the shaft, discs and bearings, the equation of motion of the whole rotor is given by

$$
\mathbf{M} \ddot{\mathbf{x}}+\left(\mathbf{C}+\omega_{r} \mathbf{G}\right) \dot{\mathbf{x}}+\mathbf{K x}=\mathbf{f}
$$

with $\mathbf{M}$ and $\mathbf{G}$ the mass and gyroscopic matrices, $\mathbf{K}$ the stiffness matrix of the shaft and bearings. $\mathbf{C}$ is the matrix of Rayleigh damping of the shaft. f corresponds to the forces applied to the system including the effects of gravity, and unbalance forces generated by faults. $\ddot{\mathbf{x}}, \dot{\mathbf{x}}$, and $\mathbf{x}$ are the acceleration, velocity and displacement degrees of freedom expressed in the rotor fixed frame. $\omega_{r}$ defines the rotational speed of the shaft.

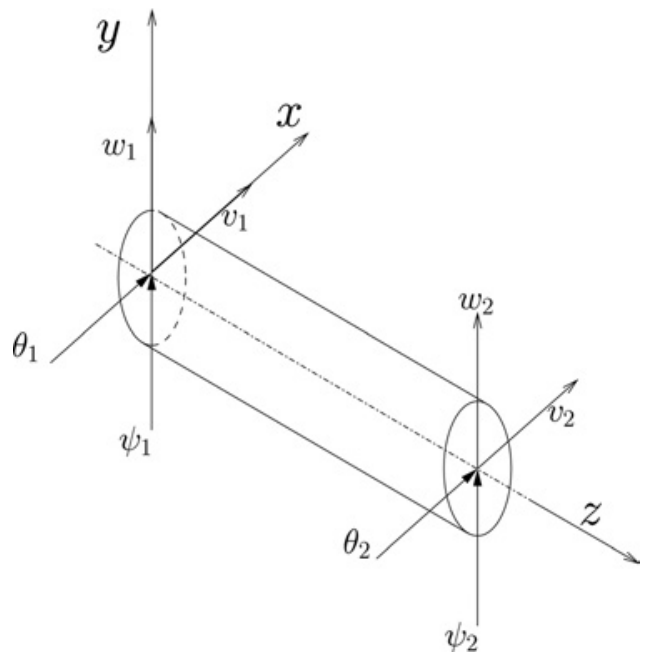

Fig. 2 Shaft finite element
2.2 Modeling Faults. In this section, we propose to present more precisely the specific models associated with excitations and asymmetry of the rotor.

2.2.1 Unbalance. The unbalance can be seen as one of the most common fault in rotating systems. In this study, this fault is present on disks and can then be modeled as adding a disk mass $m_{e}$ offset by a distance $d_{e}$ relative to the axis of rotation. Therefore this mass generates a rotating force for degrees of freedom of the $\operatorname{disk}\left[\begin{array}{llll}v & w & \theta & \psi\end{array}\right]^{T}$ that can be given by

$$
\mathbf{f}^{d}=m_{e} d_{e} \omega_{r}{ }^{2}\left[\cos \left(\omega_{r} t\right) \quad \sin \left(\omega_{r} t\right) \quad 0 \quad 0\right]^{T}
$$

2.2.2 Asymmetry of the Rotor Shaft. The asymmetry in an element of the rotor shaft is characterized by the presence of two flats on the shaft as shown in Fig. 1(b). By noting the depth of each flat with $h$, this section has the moments of inertia $I_{X}$ and $I_{Y}$ in the rotating frame $\left(X_{a}, Y_{a}, z\right)$. Expressions are given by

$$
\begin{gathered}
I_{X}=\frac{R^{4}}{2}\left(\alpha-\frac{\sin (2 \alpha)}{2}\right)+\sqrt{2 h R-h^{2}}(R-h)^{3} \\
I_{Y}=\frac{R^{4}}{2}\left(\alpha+\frac{\sin (2 \alpha)}{2}\right)+\frac{\left(2 h R-h^{2}\right)^{\frac{3}{2}}(R-h)}{3}
\end{gathered}
$$

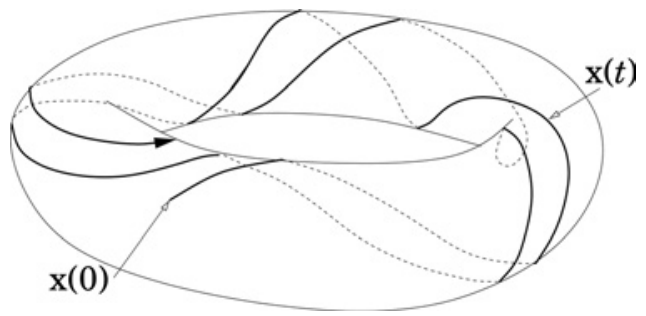

Fig. 3 Hypertime concepts related to a bi-periodic motion

Table 1 The fourth-dimensional polynomial chaoses of order 2

\begin{tabular}{lllclc}
\hline \hline$\Psi_{0}$ & 1 & $\Psi_{5}$ & $\xi_{1}^{2}-1$ & $\Psi_{10}$ & $\xi_{2} \xi_{3}$ \\
$\Psi_{1}$ & $\xi_{1}$ & $\Psi_{6}$ & $\xi_{1} \xi_{2}$ & $\Psi_{11}$ & $\xi_{2} \xi_{4}$ \\
$\Psi_{2}$ & $\xi_{2}$ & $\Psi_{7}$ & $\xi_{1} \xi_{3}$ & $\Psi_{12}$ & $\xi_{3}^{2}-1$ \\
$\Psi_{3}$ & $\xi_{3}$ & $\Psi_{8}$ & $\xi_{1} \xi_{4}$ & $\Psi_{13}$ & $\xi_{3} \xi_{4}$ \\
$\Psi_{4}$ & $\xi_{4}$ & $\Psi_{9}$ & $\xi_{2}^{2}-1$ & $\Psi_{14}$ & $\xi_{4}^{2}-1$ \\
\hline
\end{tabular}


Table 2 Example of an identification process between $\left(\xi_{1}, \xi_{2}\right)$ basis and the fourth-dimensional polynomial chaoses basis truncated to order 2

\begin{tabular}{lccccc}
\hline \hline 1 & $\Psi_{0}$ & $\xi_{1}^{2}$ & $\Psi_{5}+\Psi_{0}$ & $\xi_{2} \xi_{3}$ & $\Psi_{10}$ \\
$\xi_{1}$ & $\Psi_{1}$ & $\xi_{1} \xi_{2}$ & $\Psi_{6}$ & $\xi_{2} \xi_{4}$ & $\Psi_{11}$ \\
$\xi_{2}$ & $\Psi_{2}$ & $\xi_{1} \xi_{3}$ & $\Psi_{7}$ & $\xi_{3}^{2}$ & $\Psi_{12}+\Psi_{0}$ \\
$\xi_{3}$ & $\Psi_{3}$ & $\xi_{1} \xi_{4}$ & $\Psi_{8}$ & $\xi_{3} \xi_{4}$ & $\Psi_{13}$ \\
$\xi_{4}$ & $\Psi_{4}$ & $\xi_{2}^{2}$ & $\Psi_{9}+\Psi_{0}$ & $\xi_{4}^{2}$ & $\Psi_{14}+\Psi_{0}$ \\
\hline \hline
\end{tabular}

Table 3 Sets of parameters for the two case studies

\begin{tabular}{lllll}
\hline \hline & $\delta_{E}$ & $\delta_{U_{0}}$ & $\delta_{m}$ & $\delta_{h}$ \\
\hline Case 1 & $5 \%$ & $5 \%$ & - & - \\
Case 2 & $5 \%$ & $5 \%$ & $5 \%$ & $5 \%$ \\
\hline \hline
\end{tabular}

Table 4 Model parameters

\begin{tabular}{lc}
\hline \hline Parameters & Dimension \\
\hline Length of shaft & $1 \mathrm{~m}$ \\
Diameter of shaft & $0.04 \mathrm{~m}$ \\
Position of disc 1 & $0.6 \mathrm{~m}$ \\
Position of disc 2 & $0.8 \mathrm{~m}$ \\
Outer diameter of disc 1 & $0.2 \mathrm{~m}$ \\
Outer diameter of disc 2 & $0.4 \mathrm{~m}$ \\
Inner diameter of discs 1 and 2 & $0.04 \mathrm{~m}$ \\
Thickness of discs 1 and 2 & $0.02 \mathrm{~m}$ \\
Mean of Young's modulus $\bar{E}$ & $2.1 \times 10^{11} \mathrm{Nm}^{2}$ \\
Shear modulus $G$ & $8.0 \times 10^{10} \mathrm{Nm}^{2}$ \\
Poisson ratio $\nu$ & 0.3 \\
Density $\rho$ & $7800 \mathrm{~kg} \mathrm{~m}{ }^{-3}$ \\
Mean of mass unbalance $\overline{m_{e}}$ & $0.05 \mathrm{~kg}$ \\
Eccentricity of the mass unbalance $d_{e}$ & $0.01 \mathrm{~m}$ \\
Mean of vibration magnitude $\overline{U_{0}}$ & $1 \mathrm{~mm}$ \\
Mean of flat depth $\bar{h}$ & $2 \mathrm{~mm}$ \\
\hline \hline
\end{tabular}

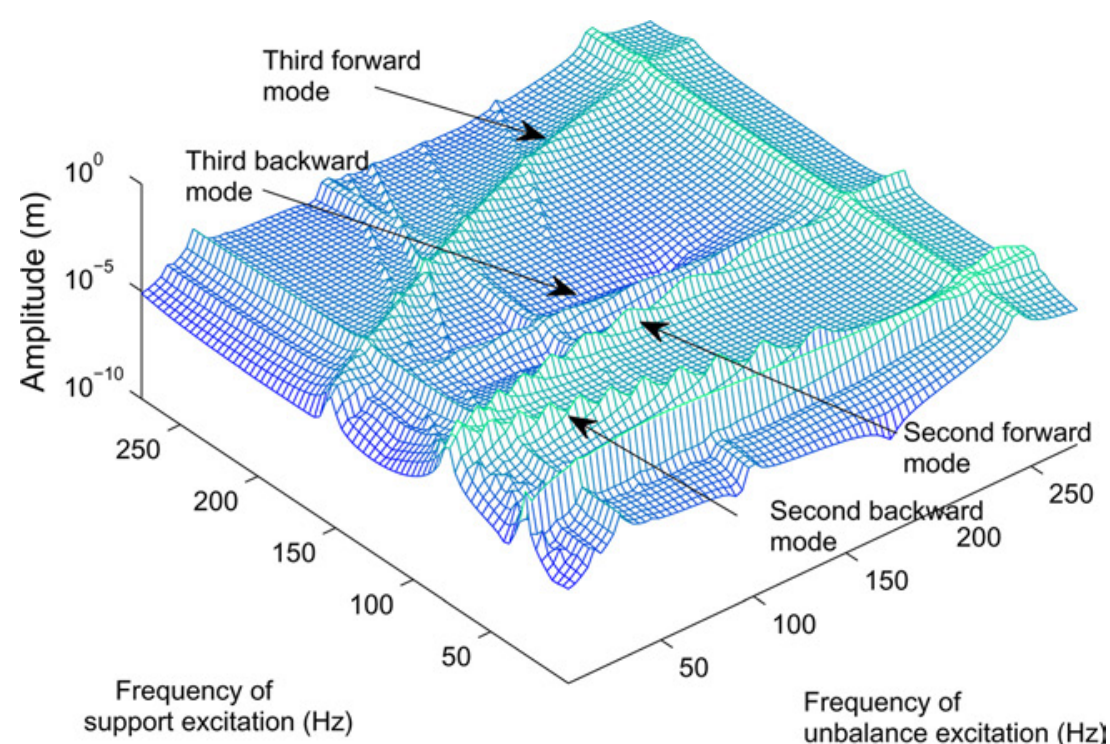

(a)

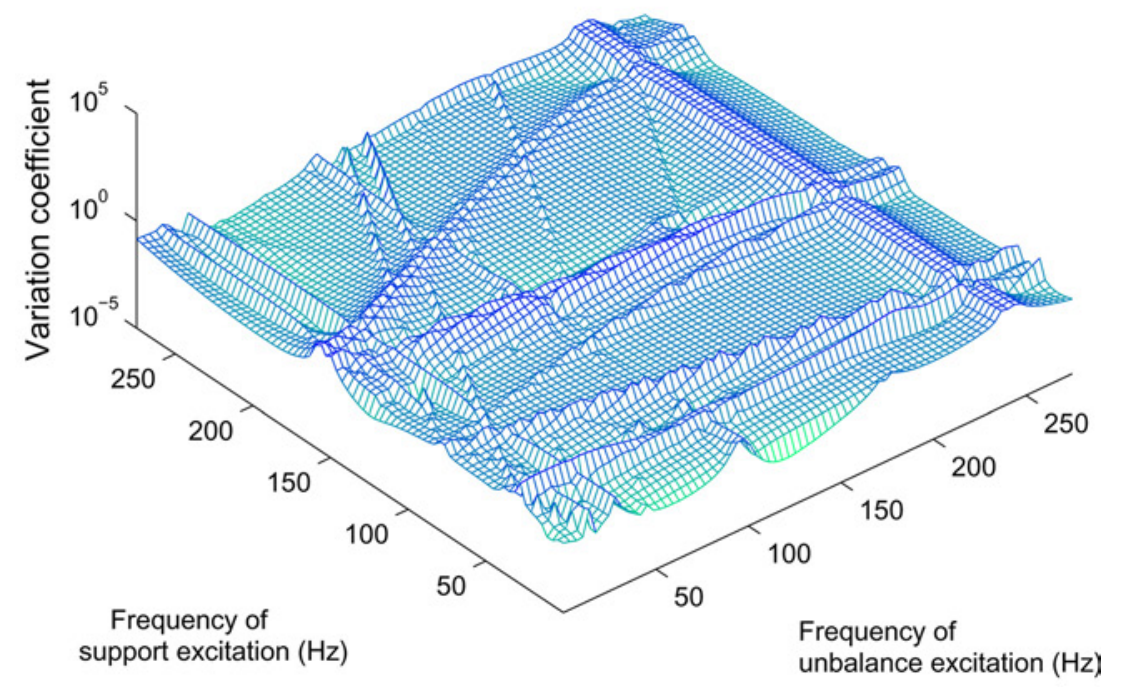

(b)

Fig. 4 Frequency Response Function via PCE (case 1): (a) mean; (b) variation coefficient 


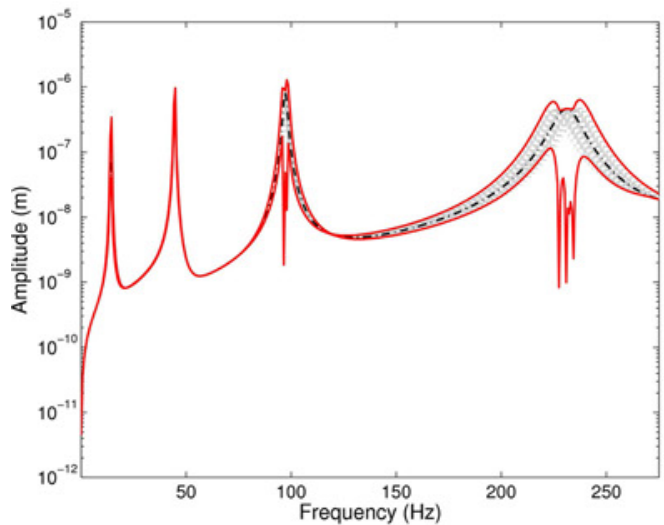

(a)

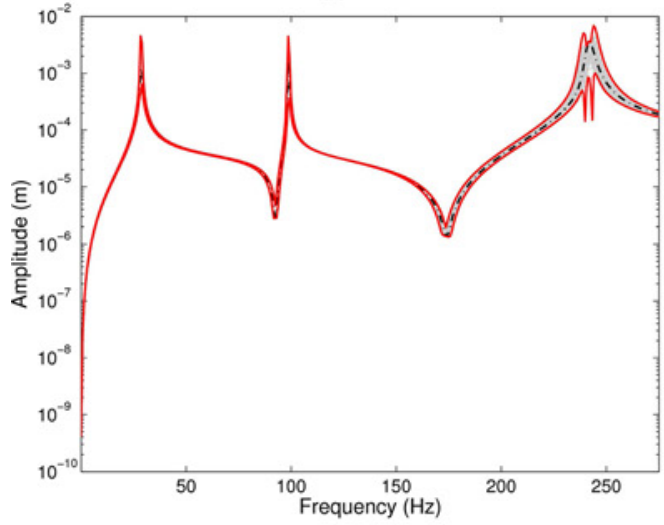

(c)

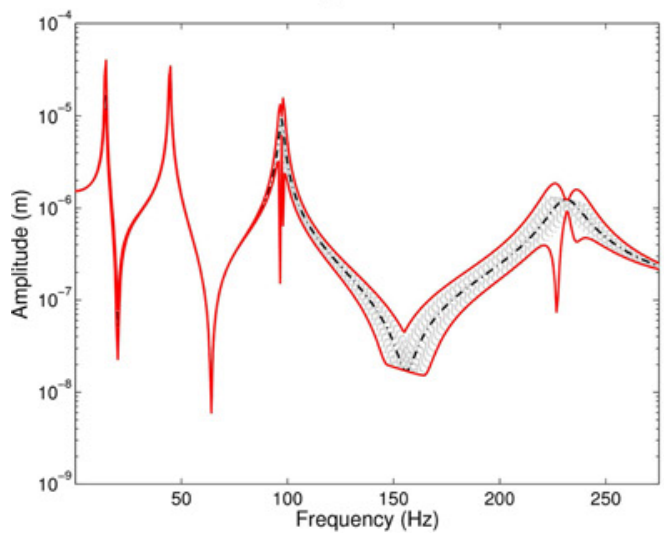

(e)

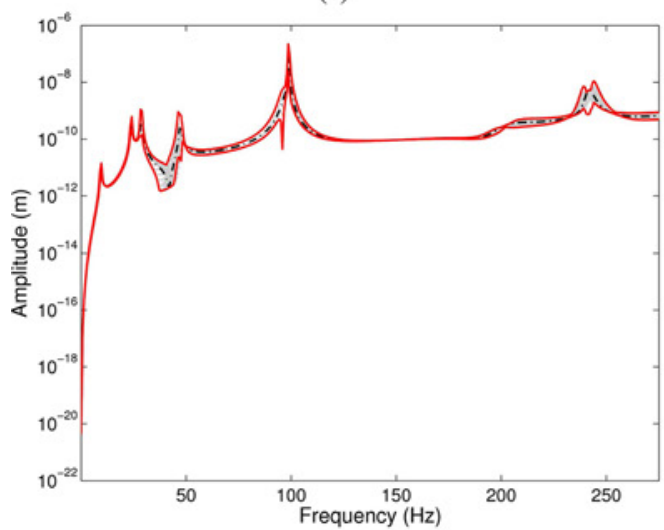

(g)

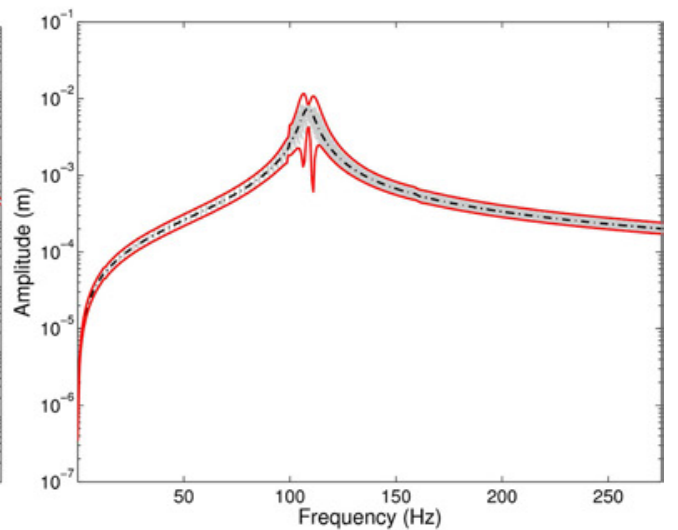

(b)

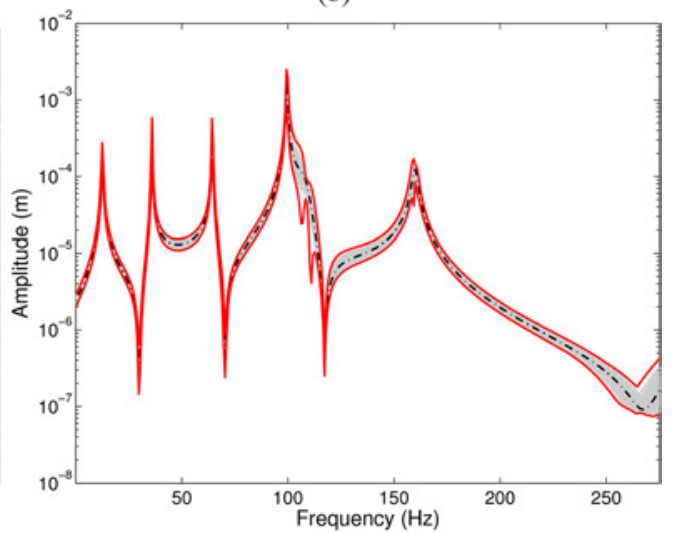

(d)

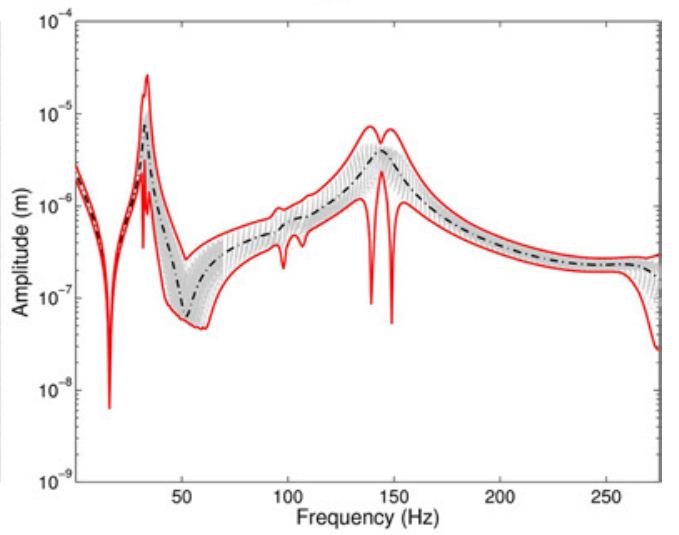

(f)

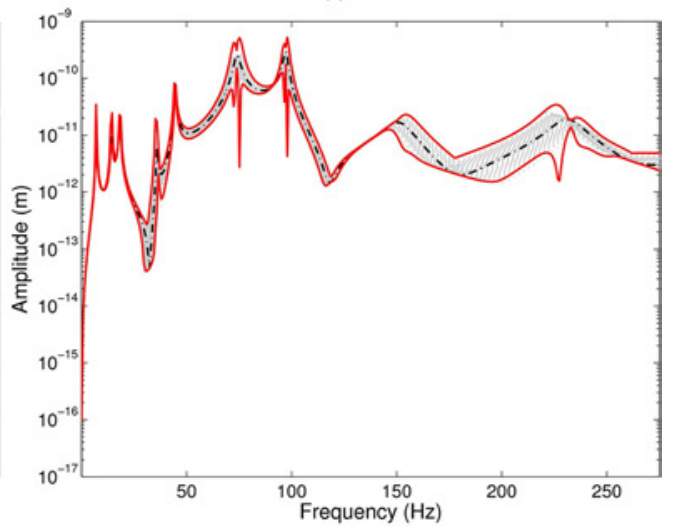

(h)

Fig. 5 Frequency Response Function (case 1 and $f_{b}=100 \mathrm{~Hz}$ ); envelope PCE (red solid lines); MCS (dotted gray lines); deterministic response (dashed-dotted black lines); order $\left[k_{1}, k_{2}\right](a)[0,0],(b)$ $[0,1],(c)[1,0],(d)[2,-1],(e)[2,0],(f)[2,1],(g)[3,0]$, and $(h)[4,0]$ 
with $\alpha$ and $R$ being the angle and the radius defining the shaft section.

Using the developments proposed by Oncescu et al. [8], the elementary matrices of mass, stiffness and gyroscopic can be expressed in the fixed frame as

$$
\begin{gathered}
\mathbf{M}^{e}=\mathbf{M t}_{0}^{e}+\mathbf{M r}_{0}^{e}+\mathbf{M}_{c}^{e} \cos \left(2 \omega_{r} t\right)+\mathbf{M}_{s}^{e} \sin \left(2 \omega_{r} t\right) \\
\mathbf{K}^{e}=\mathbf{K}_{0}^{e}+\mathbf{K}_{c}^{e} \cos \left(2 \omega_{r} t\right)+\mathbf{K}_{s}^{e} \sin \left(2 \omega_{r} t\right) \\
\mathbf{G}^{e}=\mathbf{G}_{0}^{e}
\end{gathered}
$$

Expressions $\mathbf{M t}_{0}^{e}, \mathbf{M r}_{0}^{e}, \mathbf{M}_{c}^{e}, \mathbf{M}_{s}^{e}, \mathbf{K}_{0}^{e}, \mathbf{K}_{c}^{e}, \mathbf{K}_{s}^{e}$ and $\mathbf{G}_{0}^{e}$ can be found in (Ref. [7], pages 50-56]. The mean and deviatoric moments of the area are given by $I_{m}=\frac{I_{X}+I_{Y}}{2}$ and $I_{d}=\frac{I_{X}-I_{Y}}{2}$.

2.2.3 Model of Excitation Support. As previously indicated, the support of the rotor system is subjected to an excitation in the $y$-direction with a pulsation $\omega_{b}$ at one of its bearings, as shown in Fig. 1. This excitation at the rotor base can be modeled as a force acting at the end of the shaft. It is modeled as follows:

$$
\mathbf{f}^{b}=\left[\begin{array}{llll}
0 & k_{y y} U_{0} \cos \left(\omega_{b} t\right) & 0 & 0
\end{array}\right]^{T}
$$

with $k_{y y}$ the stiffness of the bearing along $y$-direction.

2.3 Final Equation. By considering the previous developments, the overall equation of the rotor system defined by Eq. (1) can be rewritten as follows:

$$
\begin{aligned}
& \left(\mathbf{M}_{\mathbf{0}}+\mathbf{M}_{\mathbf{c}} \cos \left(2 \omega_{r} t\right)+\mathbf{M}_{\mathbf{s}} \sin \left(2 \omega_{r} t\right)\right) \ddot{\mathbf{x}}+\left(\mathbf{C}+\omega_{r} \mathbf{G}\right) \dot{\mathbf{x}} \\
& \quad+\left(\mathbf{K}_{\mathbf{0}}+\mathbf{K}_{\mathbf{c}} \cos \left(2 \omega_{r} t\right)+\mathbf{K}_{\mathbf{s}} \sin \left(2 \omega_{r} t\right)\right) \mathbf{x}=\mathbf{f}
\end{aligned}
$$

with $\mathbf{M}_{\mathbf{c}}, \mathbf{M}_{\mathbf{s}}, \mathbf{K}_{\mathbf{c}}$, and $\mathbf{K}_{\mathbf{s}}$ corresponding to the assembly for all elements of the elementary matrices of the shaft $\mathbf{M}_{c}^{e}, \mathbf{M}_{s}^{e}, \mathbf{K}_{c}^{e}$, and $\mathbf{K}_{s}^{e} . \mathbf{M}_{0}$ and $\mathbf{G}$ correspond to the assembly of the mass and gyroscopic matrices of the discs and shaft elements. Finally, $\mathbf{K}_{\mathbf{0}}$ corresponds to stiffness matrices of bearings and shaft components. $\mathbf{f}$ includes the various excitation sources.

\section{Multi-Dimensional Harmonic Balance Method with Polynomial Chaos Expansion}

In this section, we propose to describe the coupling procedure of the Multi-Dimensional Harmonic Balance Method with the Polynomial Chaos Expansion. We recall that the objective of the present study is to propose a new methodology to obtain the random quasi-periodic dynamic response of a rotor system subjected to multi-frequency excitations in the presence of uncertainty on several physical parameters.

Before presenting this approach, we begin by recalling briefly the major developments related to the Multi-Harmonic Balance Method. Then we describe the proposed methodology to combine this approach with the method of the Polynomial Chaos Expansion.

3.1 Multi-Harmonic Balance Method. In the present study, the rotor system is subjected to $p$ multiple periodic incommensurable excitation frequencies $\omega_{1}, \omega_{2}, \ldots, \omega_{p}$. The response of the system contains the frequency components of any linear combination of the incommensurable frequency components

$$
k_{1} \omega_{1}+k_{2} \omega_{2}+\ldots+k_{i} \omega_{i}+\ldots+k_{p} \omega_{p}
$$

with $k_{i}=-N_{h},-N_{h}+1, \ldots,-1,0,1, \ldots, N_{h}-1, N_{h}$ where $N_{h}$ is the order of the Fourier series.

Therefore the quasi-periodic dynamic response $\mathbf{x}(t)$ can be approximated in the form of multiple Fourier series [9]:

$$
\begin{aligned}
\mathbf{x}(t)= & \left.\sum_{k_{1}=-N_{h}}^{N_{h}} \sum_{k_{2}=-N_{h}}^{N_{h}} \ldots \sum_{k_{p}=-N_{h}}^{N_{h}} \mathbf{A}_{\left[k_{1}, k_{2}, \ldots, k_{p}\right]} \cos \sum_{i=1}^{p} k_{i} \omega_{i} t\right) \\
& \left.\left.+\mathbf{B}_{\left[k_{1}, k_{2}, \ldots, k_{p}\right]} \sin \sum_{i=1}^{p} k_{i} \omega_{i} t\right)\right)
\end{aligned}
$$

where $\mathbf{A}_{\left[k_{1}, k_{2}, \ldots, k_{p}\right]}$ and $\mathbf{B}_{\left[k_{1}, k_{2}, \ldots, k_{p}\right]}$ define the Fourier coefficients of order $\left[k_{1}, k_{2}, \ldots, k_{p}\right]$ for the quasi-periodic response $\mathbf{x}(t)$.

The previous expression can be rewritten in a condensed form:

$$
\mathbf{x}(t)=\sum_{\mathbf{k} \in \mathbb{Z}^{p}}\left(\mathbf{A}_{\mathbf{k}} \cos (\mathbf{k} \cdot \omega t)+\mathbf{B}_{\mathbf{k}} \sin (\mathbf{k} \cdot \omega t)\right)
$$

where $\omega$ is the vector of incommensurable frequencies defined by $\boldsymbol{\omega}=\left[\omega_{1}, \omega_{2}, \ldots, \omega_{p}\right] . \mathbf{k}$ is the vector containing the numbers of harmonics defined by $\mathbf{k}=\left[k_{1}, k_{2}, \ldots, k_{p}\right]$ with $k_{i}=-N_{h},-N_{h}+1, \ldots$, $-1,0,1, \ldots, N_{h}-1, N_{h}$ for $i=1, \ldots, p$ and ${ }_{i=1}^{p}\left|k_{i}\right| \leq N_{h}$. So, $\mathbf{A}_{\mathbf{k}}$ and $\mathbf{B}_{\mathbf{k}}$ define the unknown Fourier coefficients of any linear combinations of the incommensurable frequency components or the quasi-periodic response of the rotor system.

Then, it can be observed that the global excitation $\mathbf{f}(t)$ can also be expressed in the following form:

$$
\mathbf{f}(t)=\sum_{\mathbf{k} \in \mathbb{Z}^{p}}\left(\mathbf{S}_{\mathbf{k}} \cos (\mathbf{k} \cdot \omega t)+\mathbf{T}_{\mathbf{k}} \sin (\mathbf{k} \cdot \omega t)\right)
$$

In this kind of problem, the concept of multi-dimensional time domain can be used by considering an equivalent function $\hat{\mathbf{x}}(t)$ of $p$ time variables in a $p$-dimensional time domain $\mathbf{T}=\left[T_{1}, \ldots, T_{p}\right]=\omega t, 2 \pi$ periodic on each component of the hyper-time:

$$
\mathbf{x}(t)=\hat{\mathbf{x}}(\mathbf{T})=\hat{\mathbf{x}}(\omega t)
$$

In the present study, the global excitation has two incommensurable frequencies $\omega_{r}$ and $\omega_{b}$. Therefore $\boldsymbol{\omega}=\left[\omega_{r}, \omega_{b}\right]$ and $\mathbf{k}=\left[k_{1}, k_{2}\right]$. We denote $m$ the dimension of the basis. As such, the bi-periodic response $\mathbf{x}(t)$ covers the invariant torus in the phase space, as illustrated in Fig. 3.

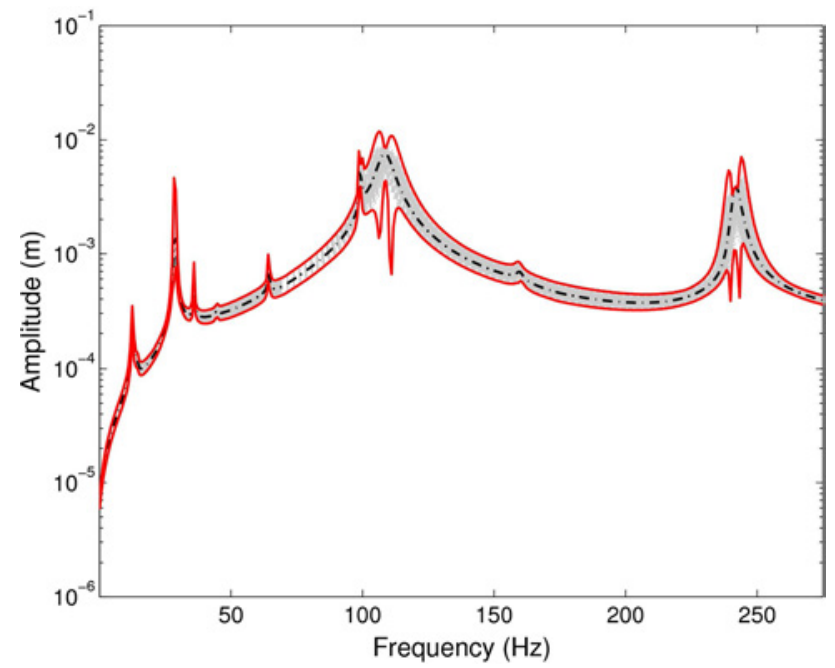

Fig. 6 Frequency Response Function (case 1 and $f_{b}=100 \mathrm{~Hz}$ ), global quasi-periodic response, envelope PCE (red solid lines), MCS (dotted gray lines), deterministic response (dashed-dotted black line) 

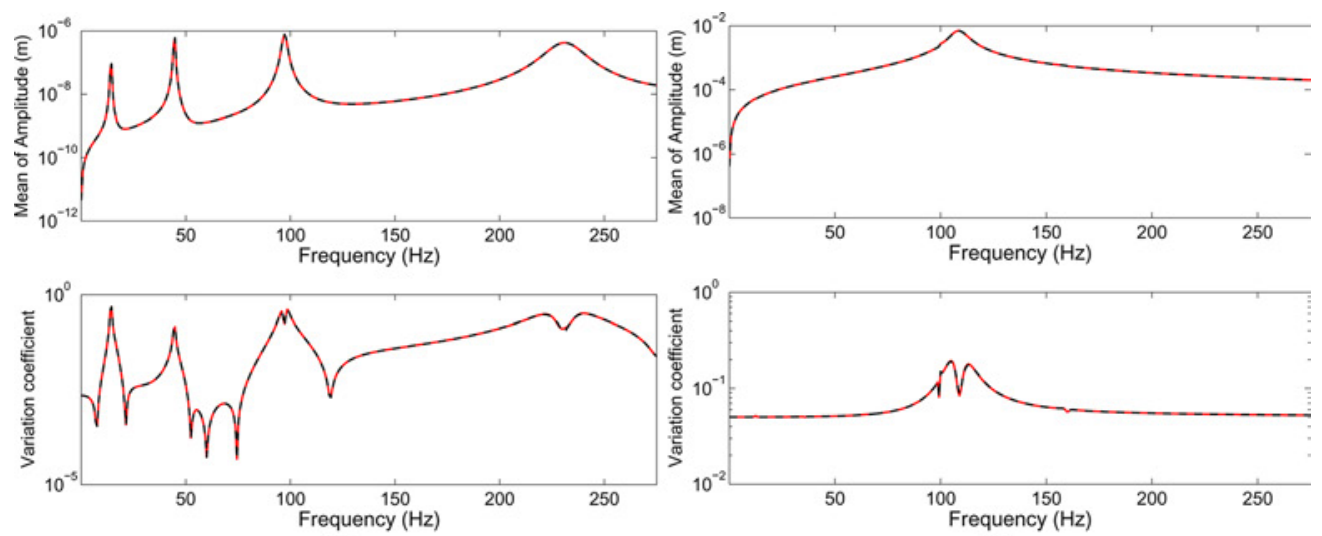

(a)

(b)
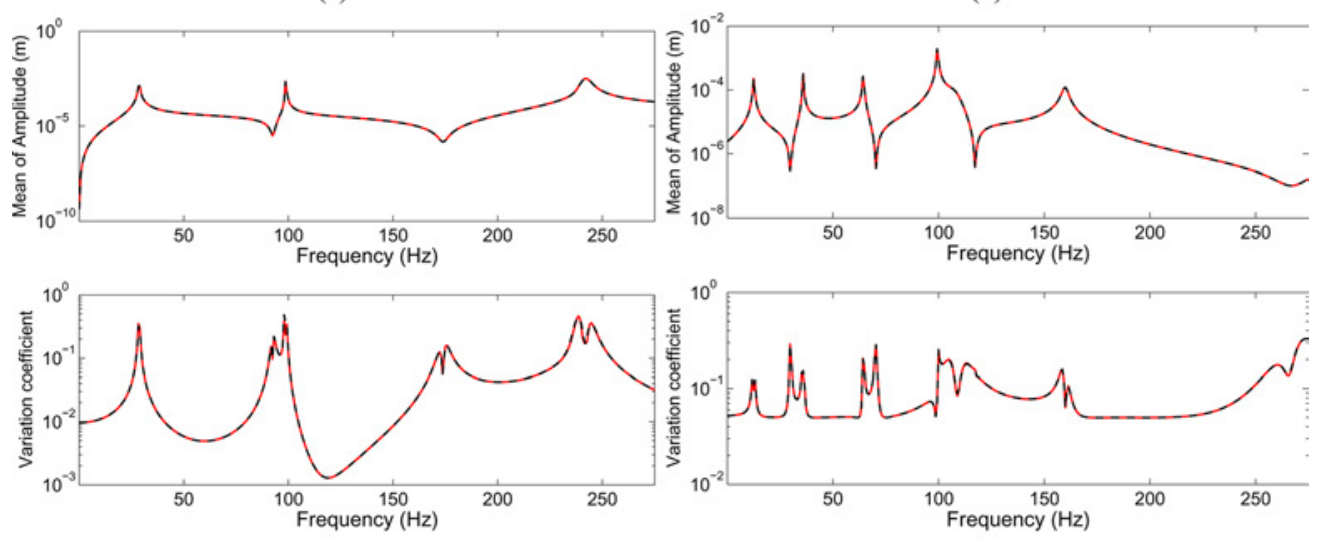

(c)

(d)
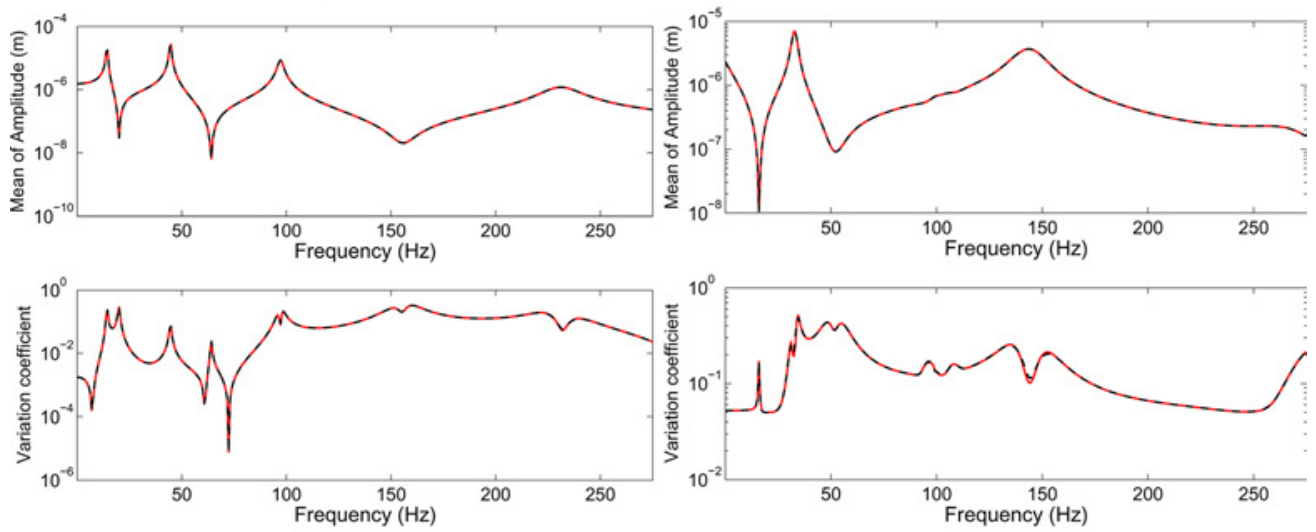

(e)

(f)
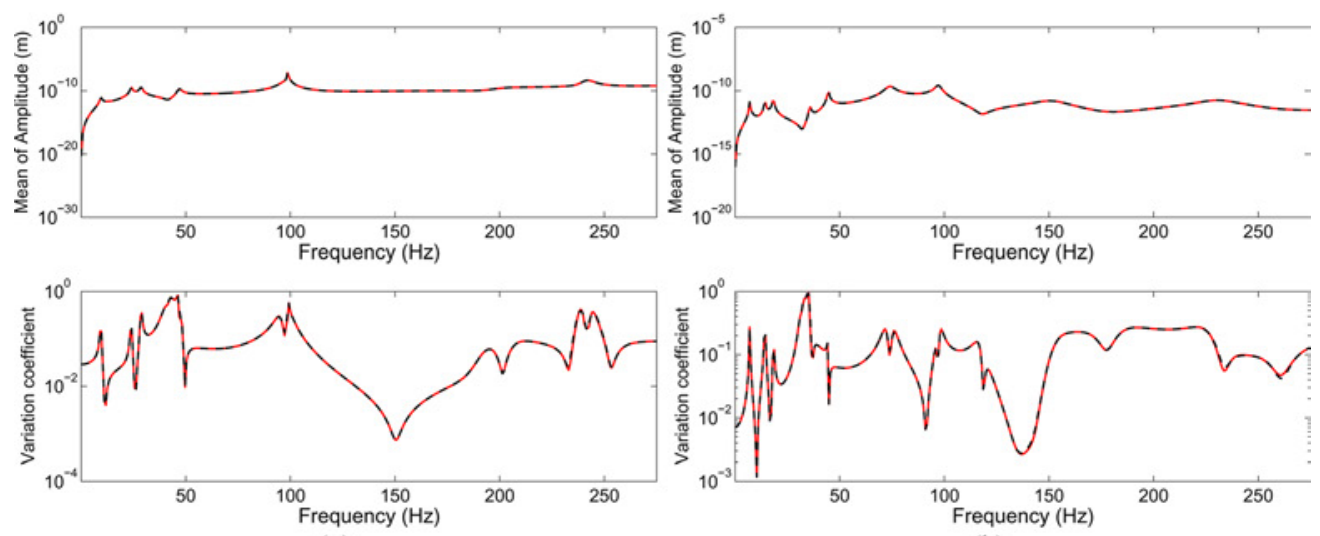

(g)

(h)

Fig. 7 Mean and variance of the FRFs (case 1 and $f_{b}=100 \mathrm{~Hz}$ ), PCE (red solid lines), MCS (dashed black lines), order FRFs $\left[k_{1}, k_{2}\right](a)[0,0],(b)[0,1],(c)[1,0],(d)[2,-1],(e)[2,0],(f)[2,1],(g)[3,0],(h)$ $[4,0]$

6 
Substituting the expressions in Eqs. (12)-(13) in the equation of motion of the system (Eq. (9)), after projection, the rotor dynamic equation is written as a linear system of dimension $(2 m+1) n_{d d l}$ with $n_{d d l}$ being the number of degrees of freedom:

$$
\mathbf{L X}=\mathbf{f}
$$

with $\mathbf{L}, \mathbf{X}$ and $\mathbf{f}$ defined by

$$
\mathbf{L}=\mathbf{I}_{1}+\mathbf{I}_{2}+\mathbf{I}_{3}
$$

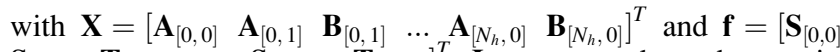
$\begin{array}{lllll}\mathbf{S}_{[0,1]} & \mathbf{T}_{[0,1]} & \ldots & \mathbf{S}_{\left[N_{h}, 0\right]} & \left.\mathbf{T}_{\left[N_{h}, 0\right]}\right]^{T} . \mathbf{I}_{1} \text { corresponds to the rewrit- }\end{array}$ ing of the constant terms in the form of a linear system, $\mathbf{I}_{2}$ corresponds to the parametric terms due to the stiffness contribution and $\mathbf{I}_{3}$ is the parametric terms due to the mass contribution from Eq. (9). The various expressions of $\mathbf{I}_{1}, \mathbf{I}_{2}$, and $\mathbf{I}_{3}$ are given in Appendix A.

3.2 Stochastic Expression of the Problem. As previously explained, some excitations and physical parameters of material or geometrical types can be taken as random. Denoting by $\tau$ the random character of one variable, we define the new random mass, damping, gyroscopic, stiffness matrices $\tilde{\mathbf{M}}(\tau), \tilde{\mathbf{C}}(\tau), \tilde{\mathbf{G}}(\tau)$, $\tilde{\mathbf{K}}(\tau)$ and the new random excitation force vector $\tilde{\mathbf{f}}(\tau)$. Details on each model for these random quantities will be given in the following sections, however, each random physical quantity $\tilde{\mathbf{a}}(\tau)$ is expanded on the Karhunen-Loeve expansion with the Galerkin formulation of the finite element method (See Ref. [5], pages 13-92) as follows:

$$
\tilde{\mathbf{a}}(\tau)=\overline{\mathbf{a}}+\sum_{l=1}^{L} \xi_{l}(\tau) \mathbf{a}_{l}
$$

where $\left\{\xi_{1}(\tau), \ldots \xi_{L}(\tau)\right\}$ consists in an orthonormal basis of Gaussian random variables, $\overline{\mathbf{a}}$ designates the mean of quantity $\tilde{\mathbf{a}}$ and $\mathbf{a}_{l}$ is the $l$ th term of the Karhunen-Loeve expansion. Then, Eq. (16) can be rewritten in a random way such as

$$
\tilde{\mathbf{L}}(\tau)=\tilde{\mathbf{I}}_{1}(\tau)+\tilde{\mathbf{I}}_{2}(\tau)+\tilde{\mathbf{I}}_{3}(\tau)
$$

which yields finally to a random dynamical response $\tilde{\mathbf{X}}$ (since it verified Eq. (15)) that verifies

$$
\tilde{\mathbf{L}}(\tau) \tilde{\mathbf{X}}(\tau)=\tilde{\mathbf{f}}(\tau)
$$

One random expression of $\tilde{\mathbf{X}}$ can be an expansion on the Polynomial Chaos basis such as:

$$
\tilde{\mathbf{X}}(\tau)=\sum_{j=0}^{\infty} \mathbf{X}_{j} \Psi_{j}(\underline{\xi}(\tau))
$$

where $\Psi_{j}(\xi(\tau))$ are the multi-dimensional Hermite polynomials and $\mathbf{X}_{j}$ the $j$ th unknown deterministic vector associated to polyno$\operatorname{mial} \Psi_{j}(\underline{\xi}(\tau))[5,10]$.

Substituting Eq. (20) in Eq. (19), the system of equations to be solved is:

$$
\sum_{j=0}^{\infty} \tilde{\mathbf{L}}(\tau) \mathbf{X}_{j} \Psi_{j}(\underline{\xi}(\tau))=\tilde{\mathbf{f}}(\tau)
$$

where expressions of $\tilde{\mathbf{L}}(\tau)$ and $\tilde{\mathbf{f}}(\tau)$ can be rewritten on the Polynomial Chaos basis, using Eqs. (17) and (18), such as

$$
\tilde{\mathbf{L}}(\tau)=\sum_{i=0}^{\infty} \mathbf{L}_{i} \Psi_{i}(\underline{\xi}(\tau)), \quad \tilde{\mathbf{f}}(\tau)=\sum_{j=0}^{\infty} \mathbf{f}_{i} \Psi_{i}(\underline{\xi}(\tau))
$$

Coefficients $\mathbf{L}_{i}$ will be found following one identification process obtained from the expressions of $\tilde{\mathbf{I}}_{1}(\tau), \tilde{\mathbf{I}}_{2}(\tau)$ and $\tilde{\mathbf{I}}_{3}(\tau)$ and their summation (see future sections for details). A similar identification process will be done to find $\mathbf{f}_{i}$ from the various excitation terms. In the following section of the paper, dependence on random character $\tau$ or random variable $\xi$ will be omitted. Finally, Eq. (19), written on the Polynomial Chaos basis, is given by

$$
\left.\left.\sum_{i=0}^{\infty} \mathbf{L}_{i} \Psi_{i}\right) \quad \sum_{j=0}^{\infty} \mathbf{X}_{j} \Psi_{j}\right)=\sum_{j=0}^{\infty} \mathbf{f}_{j} \Psi_{j}
$$

Solving Eq. (23) needs to project it on subset $\left\{\Psi_{k}\right\}_{k=0}^{\infty}$. Generally, to compute, it is possible to truncate the infinite basis to a $P$ order finite basis; $P$ is the number of Hermite polynomials and is defined by $P=\frac{(p+r) !}{p ! r !}$, where $r$ is the number of random variables and $p$ the chaos order. The linear system to be solved is then given by

$$
\hat{\mathbf{L}} \hat{\mathbf{X}}=\hat{\mathbf{f}}
$$

of components :

$$
\begin{gathered}
\hat{\mathbf{L}}_{j k}=\sum_{i=0}^{P} E\left\{\Psi_{i} \Psi_{j} \Psi_{k}\right\} \mathbf{L}_{i} \quad j, k=0, \ldots, P \\
\hat{\mathbf{X}}=\left[\begin{array}{lllll}
\mathbf{X}_{0} & \ldots & \mathbf{X}_{P}
\end{array}\right]^{T}, \quad \hat{\mathbf{f}}=\left[\begin{array}{lll}
\mathbf{f}_{0} E\left\{\Psi_{0}^{2}\right\} & \ldots & \mathbf{f}_{P} E\left\{\Psi_{P}^{2}\right\}
\end{array}\right]^{T}
\end{gathered}
$$

where $E\{\}$ is the mathematical expectation.

3.3 Uncertainties on the Rotor Shaft. Uncertainties on the rotor shaft can be due to uncertainties on the Young's modulus $E$ and on the geometrical parameter $h$ which then have to be modeled randomly. Using Eq. (17), we have, respectively,

$$
\tilde{E}(\tau)=\bar{E}\left(1+\delta_{E} \xi_{1}\right)
$$
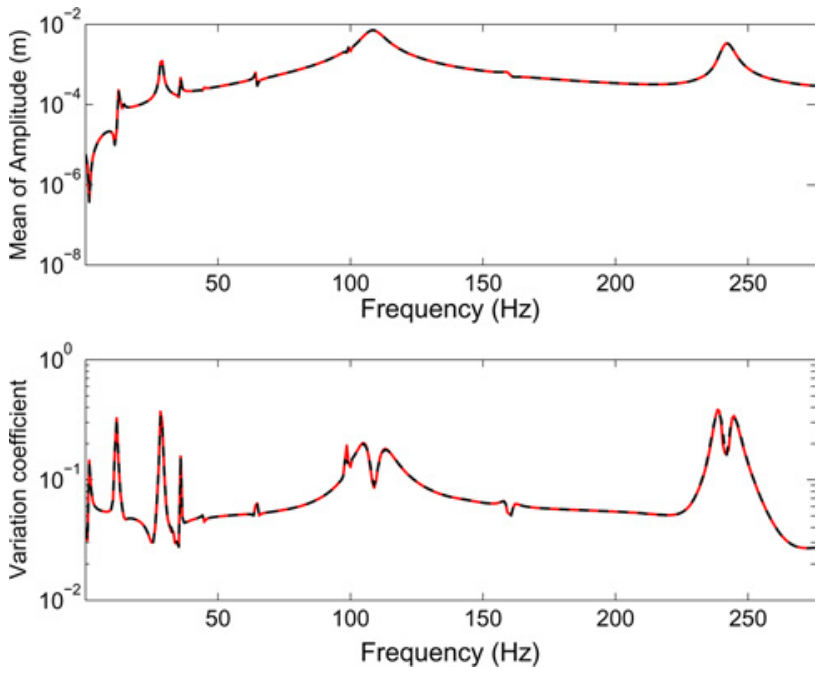

Fig. 8 Mean and variance of the FRFs (case 1 and $f_{b}=100 \mathrm{~Hz}$ ), global quasi-periodic response, PCE (red solid lines), MCS (dashed black lines) 


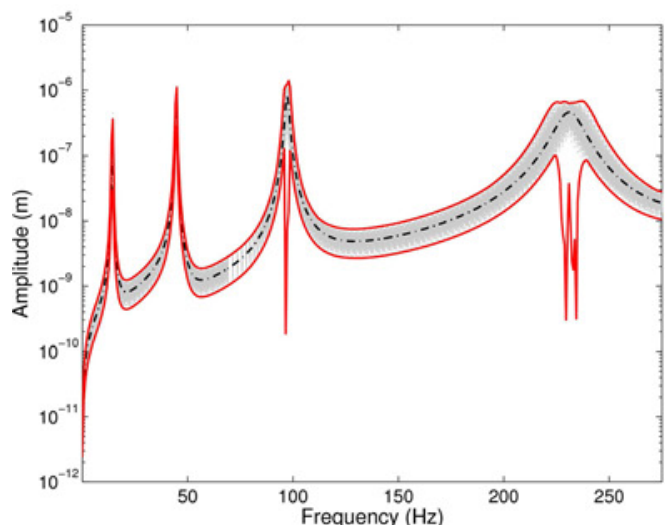

(a)

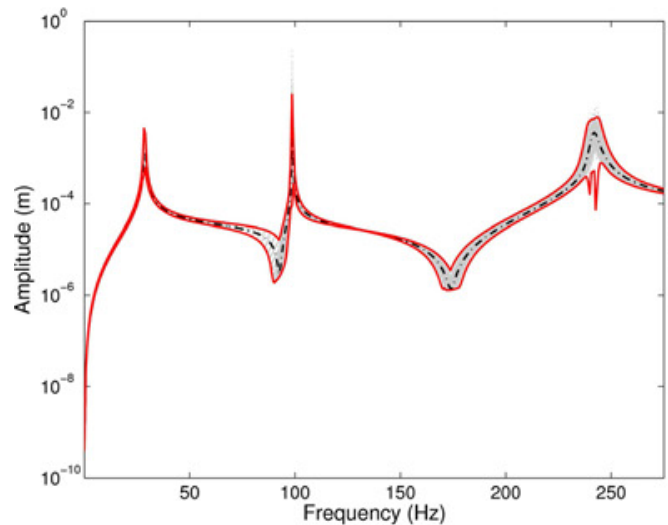

(c)

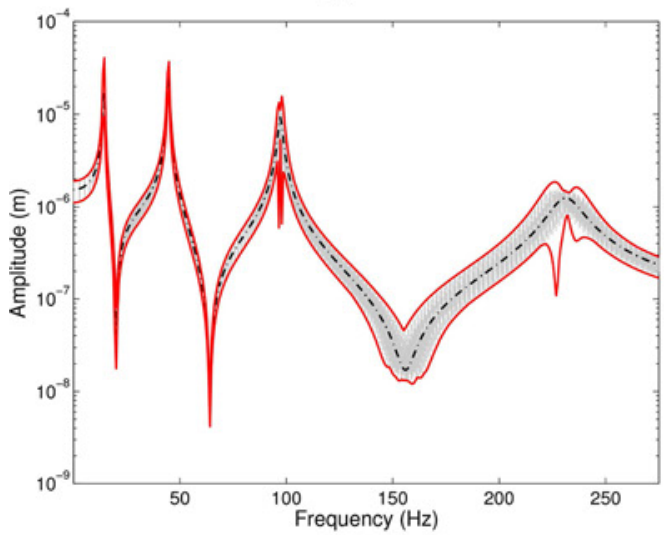

(e)

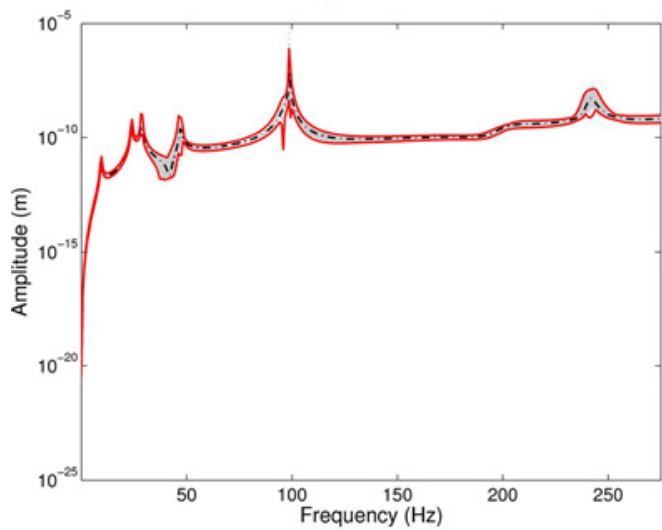

(g)

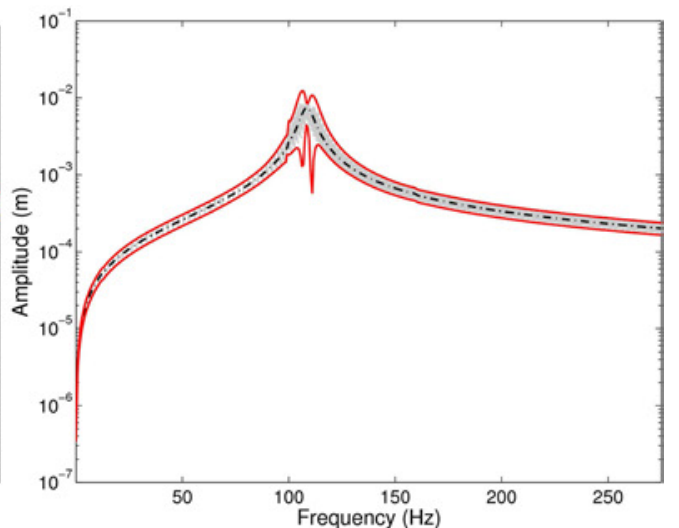

(b)

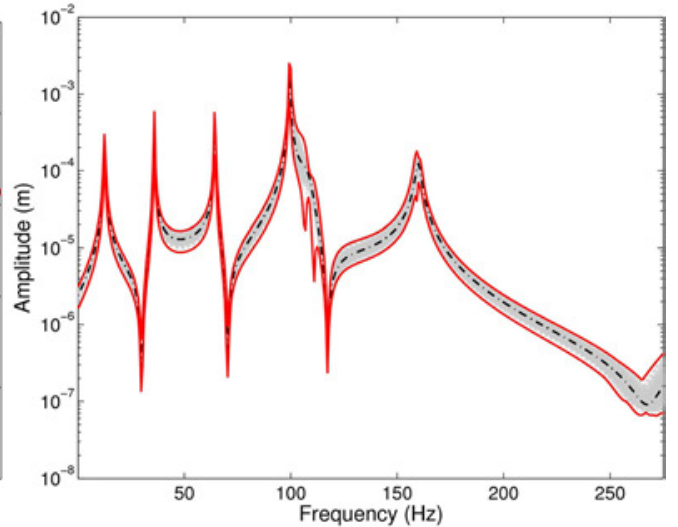

(d)

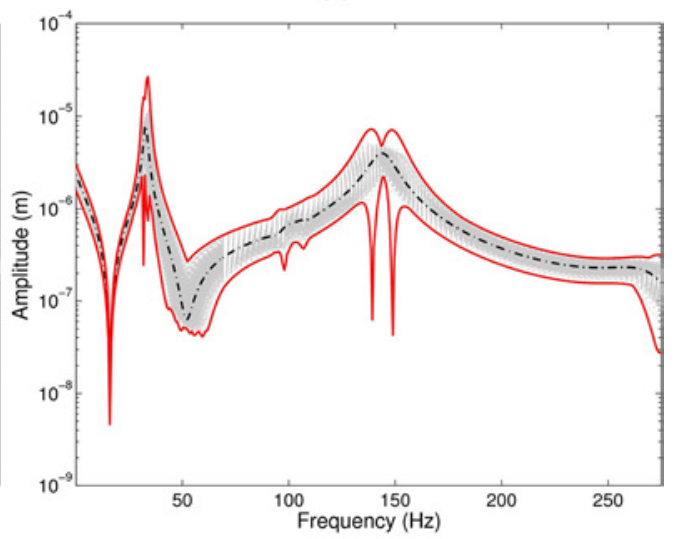

(f)

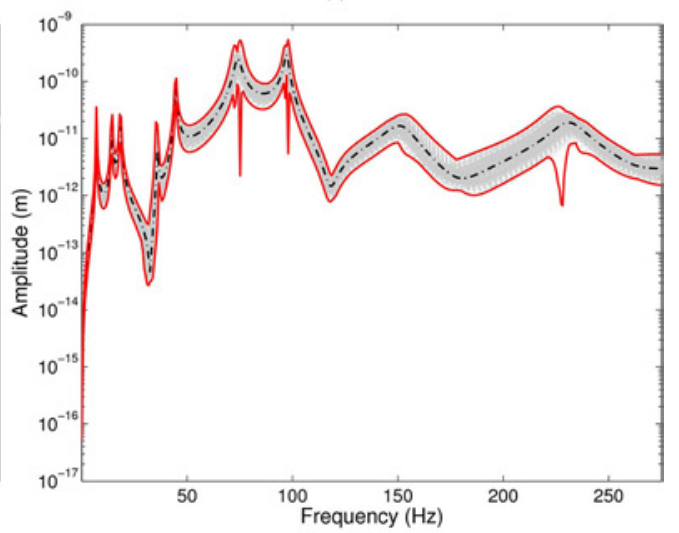

(h)

Fig. 9 Frequency Response Function (case 2 and $f_{b}=100 \mathrm{~Hz}$ ); envelope PCE (red solid lines), MCS (dotted gray lines), deterministic response (dashed-dotted black lines); order $\left[k_{1}, k_{2}\right](a)[0,0],(b)$ $[0,1],(c)[1,0],(d)[2,-1],(e)[2,0],(f)[2,1],(g)[3,0]$, and $(h)[4,0]$ 


$$
\tilde{h}(\tau)=\bar{h}\left(1+\delta_{h} \xi_{2}\right)
$$

where $\xi_{1}$ and $\xi_{2}$ are two Gaussian variables, $\delta_{a}$ designates the variation coefficient of random quantity $\tilde{a}$. Let $\tilde{h}(\tau)=\bar{h}+h^{\prime}(\tau)$, since $h^{\prime}$ is small compare to $\bar{h}$, the random section presented in Fig. 1(b) can be reduced to a rectangular section of height $h^{\prime}$. Consequently, quadratic moments $\tilde{I}_{X}(\tau)$ and $\tilde{I}_{Y}(\tau)$, random, are defined by:

$$
\tilde{I}_{X}(\tau)=\overline{I_{X}}+\frac{b h^{\prime 3}}{6}+2 b h^{\prime}\left(R-\bar{h}-\frac{h^{\prime}}{2}\right), \quad \tilde{I}_{Y}(\tau)=\overline{I_{Y}}+\frac{h^{\prime} b^{3}}{6}
$$

where

$$
b=2 \sqrt{2 \bar{h} R-\bar{h}^{2}}
$$

Besides, the mean of the quadratic moments $\overline{I_{X}}$ and $\overline{I_{Y}}$ are obtained directly from Eqs. (3)-(4). After calculations, we obtain

$$
\tilde{I}_{X}(\tau)=\sum_{i=0}^{3} \tilde{I}_{X_{i}} \xi_{2}^{i}, \quad \tilde{I}_{Y}(\tau)=\sum_{i=0}^{1} \tilde{I}_{Y_{i}} \xi_{2}^{i}
$$

with

$$
\begin{gathered}
\tilde{I}_{X_{0}}=I_{X}, \quad \tilde{I}_{X_{1}}=2 b \bar{h}(R-\bar{h}) \delta_{h}, \quad \tilde{I}_{X_{2}}=-b \bar{h}^{2} \delta_{h}{ }^{2}, \\
\tilde{I}_{X_{3}}=\frac{1}{6} b \bar{h}^{3} \delta_{h}{ }^{3} \\
\tilde{I}_{Y_{0}}=I_{Y}, \quad \tilde{I}_{Y_{1}}=\frac{1}{6} \bar{h} \delta_{h} b^{3}
\end{gathered}
$$

Thus, the mean and deviatoric quadratic moments are stochastic quantities and we can easily deduce their expression, as a function of powers of $\xi_{2}$, such as

$$
\begin{aligned}
& \tilde{I}_{m}(\tau)=\sum_{i=0}^{3}\left(\tilde{I}_{X_{i}}+\tilde{I}_{Y_{i}}\right) \xi_{2}^{i}=\sum_{i=0}^{3} \tilde{I}_{m_{i}} \xi_{2}^{i} \\
& \tilde{I}_{d}(\tau)=\sum_{i=0}^{3}\left(\tilde{I}_{X_{i}}-\tilde{I}_{Y_{i}}\right) \xi_{2}^{i}=\sum_{i=0}^{3} \tilde{I}_{d_{i}} \xi_{2}^{i}
\end{aligned}
$$

The expressions of $\tilde{E}, \tilde{I}_{m}$, and $\tilde{I}_{d}$ (see Eqs. (27), (34), (35)), introduced in the deterministic expressions of the elementary matrices $\mathbf{K}_{0}^{e}, \mathbf{K}_{c}^{e}$, and $\mathbf{K}_{s}^{e}$ also valid in the stochastic domain, yield to expressions of random elementary matrices of rigidity $\mathbf{K}_{0}^{e}, \tilde{\mathbf{K}}_{c}^{e}$, and $\tilde{\mathbf{K}}_{s}^{e}$ as functions of $\xi_{1}$ and $\xi_{2}$

$$
\begin{aligned}
\tilde{\mathbf{K}}_{0}^{e} & =\sum_{i=0}^{3} \sum_{j=0}^{1} \tilde{\mathbf{K}}_{0_{i j}}^{e} \xi_{1}^{j} \xi_{2}^{i}, \quad \tilde{\mathbf{K}}_{c}^{e}=\sum_{i=0}^{3} \sum_{j=0}^{1} \tilde{\mathbf{K}}_{c_{i j}}^{e} \xi_{1}^{j} \xi_{2}^{i}, \\
\tilde{\mathbf{K}}_{s}^{e} & =\sum_{i=0}^{3} \sum_{j=0}^{1} \tilde{\mathbf{K}}_{s_{i j}}^{e} \xi_{1}^{j} \xi_{2}^{i}
\end{aligned}
$$

with

$$
\tilde{\mathbf{K}}_{0_{i j}}^{e}=\bar{E} \delta_{E}^{j} \tilde{I}_{m_{i}} \frac{\mathbf{K}_{0}^{e}}{E I_{m}}, \quad \tilde{\mathbf{K}}_{c_{i j}}^{e}=\bar{E} \delta_{E}^{j} \tilde{I}_{d_{i}} \frac{\mathbf{K}_{c}^{e}}{E I_{d}}, \quad \tilde{\mathbf{K}}_{s_{i j}}^{e}=\bar{E} \delta_{E}^{j} \tilde{I}_{d_{i}} \frac{\mathbf{K}_{s}^{e}}{E I_{d}}
$$

After assembling matrices $\tilde{\mathbf{K}}_{0_{i j}}^{e}, \tilde{\mathbf{K}}_{c_{i j}}^{e}, \tilde{\mathbf{K}}_{s_{i j}}^{e}$ and stiffness matrices of supports, the random matrices of rigidity for the whole structure are given by :

$$
\begin{gathered}
\tilde{\mathbf{K}}_{0}=\sum_{i=0}^{3} \sum_{j=0}^{1} \tilde{\mathbf{K}}_{0_{i j}} \xi_{1}^{j} \xi_{2}^{i}, \quad \tilde{\mathbf{K}}_{c}=\sum_{i=0}^{3} \sum_{j=0}^{1} \tilde{\mathbf{K}}_{c_{i j}} \xi_{1}^{j} \xi_{2}^{i}, \\
\tilde{\mathbf{K}}_{s}=\sum_{i=0}^{3} \sum_{j=0}^{1} \tilde{\mathbf{K}}_{s_{i j}} \xi_{1}^{j} \xi_{2}^{i}
\end{gathered}
$$

Finally, these expressions can be rewritten on the chaos basis, of random vector $\xi(\tau)$, by using the relations between the random variables and the Hermite polynomials (see Tables 1 and 2 for the case of a fourth-dimensional polynomial chaoses problem with order 2) :

$$
\tilde{\mathbf{K}}_{0}=\sum_{j=0}^{P} \tilde{\tilde{\mathbf{K}}}_{0_{j}} \Psi_{j}, \quad \tilde{\mathbf{K}}_{c}=\sum_{j=0}^{P} \tilde{\tilde{\mathbf{K}}}_{c_{j}} \Psi_{j}, \quad \tilde{\mathbf{K}}_{s}=\sum_{j=0}^{P} \tilde{\tilde{\mathbf{K}}}_{s_{j}} \Psi_{j}
$$

where coefficients $\tilde{\tilde{\mathbf{K}}}_{0_{j}}, \tilde{\tilde{\mathbf{K}}}_{c_{j}}$, and $\tilde{\tilde{\mathbf{K}}}_{s_{j}}$ are identified from relations in Table 2 with Table 1 . Since $\tilde{I}_{m}(\tau)$ and $\tilde{I}_{d}(\tau)$ are also present in the mass and gyroscopic matrices, we can similarly obtain

$$
\begin{aligned}
& \tilde{\mathbf{G}}^{e}=\sum_{i=0}^{3} \tilde{\mathbf{G}}_{i}^{e} \xi_{2}^{i}, \quad \tilde{\mathbf{M}} \mathbf{r}_{0}^{e}=\sum_{i=0}^{3} \tilde{\mathbf{M}} \tilde{r}_{0_{i}}^{e} \xi_{2}^{i}, \quad \tilde{\mathbf{M}}_{c}^{e}=\sum_{i=0}^{3} \tilde{\mathbf{M}}_{c_{i}}^{e} \xi_{2}^{i}, \\
& \tilde{\mathbf{M}}_{s}^{e}=\sum_{i=0}^{3} \tilde{\mathbf{M}}_{s_{i}}^{e} \xi_{2}^{i}
\end{aligned}
$$

where

$\tilde{\mathbf{G}}_{i}^{e}=\frac{\tilde{I}_{m_{i}}}{I_{m}} \mathbf{G}^{e}, \quad \tilde{\mathbf{M r}}_{0_{i}}^{e}=\frac{\tilde{I}_{m_{i}}}{I_{m}} \mathbf{M r}_{0}^{e}, \quad \tilde{\mathbf{M}}_{c_{i}}^{e}=\frac{\tilde{I}_{d_{i}}}{I_{d}} \mathbf{M}_{c}^{e}, \quad \tilde{\mathbf{M}}_{s_{i}}^{e}=\frac{\tilde{I}_{d_{i}}}{I_{d}} \mathbf{M}_{s}^{e}$

After the assembling process and the projection on the chaos basis, the mass and gyroscopic matrices are given by

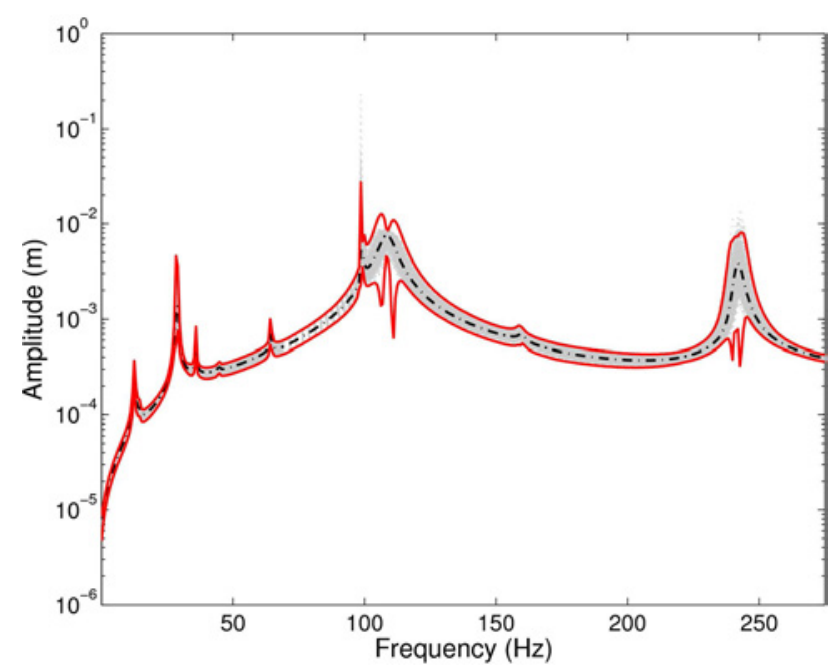

Fig. 10 Frequency Response Function (case 2 and $f_{b}=100$ $\mathrm{Hz}$ ); global quasi-periodic response, envelope PCE (red solid lines), MCS (dotted gray lines), deterministic response (dasheddotted black line) 


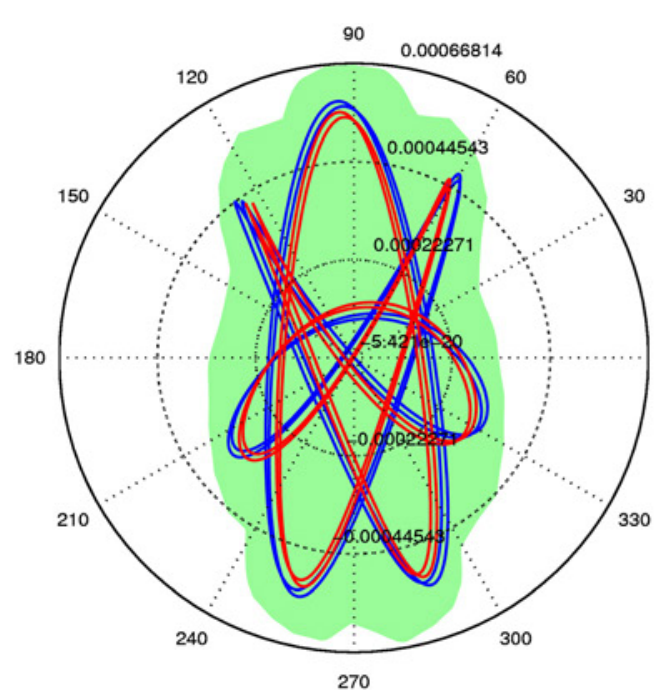

(a)

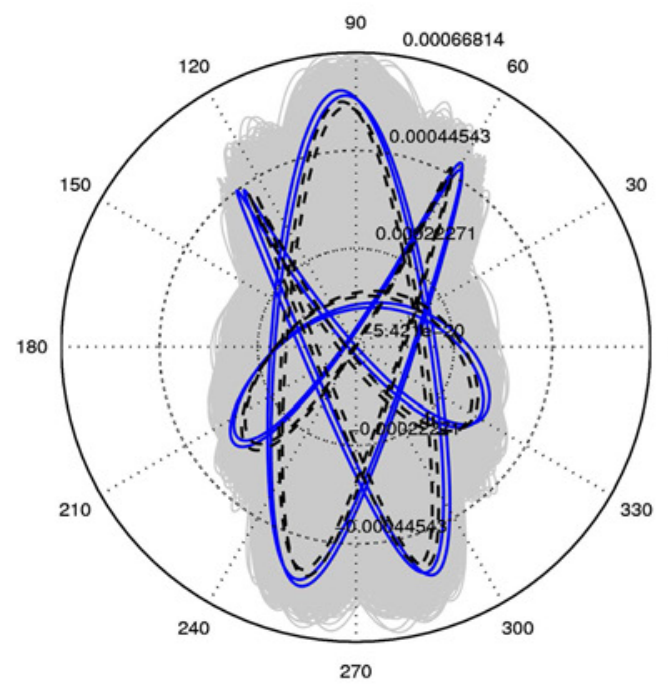

(c)

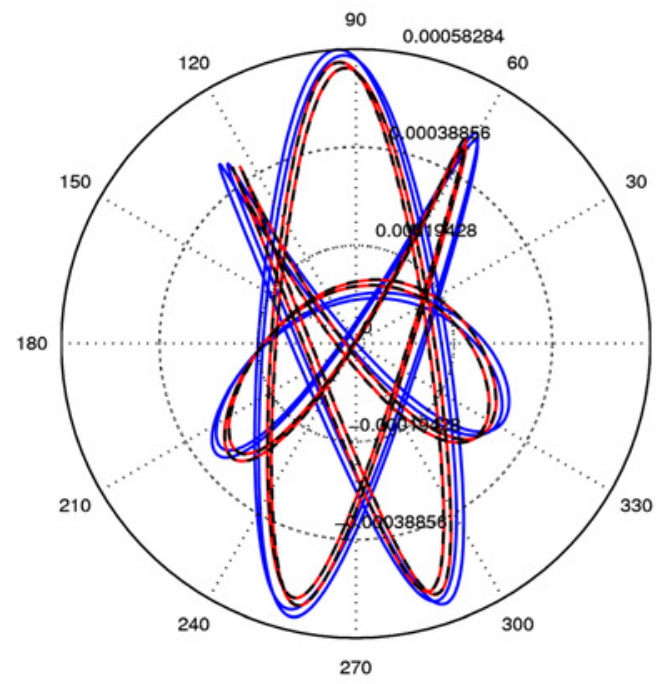

(e)

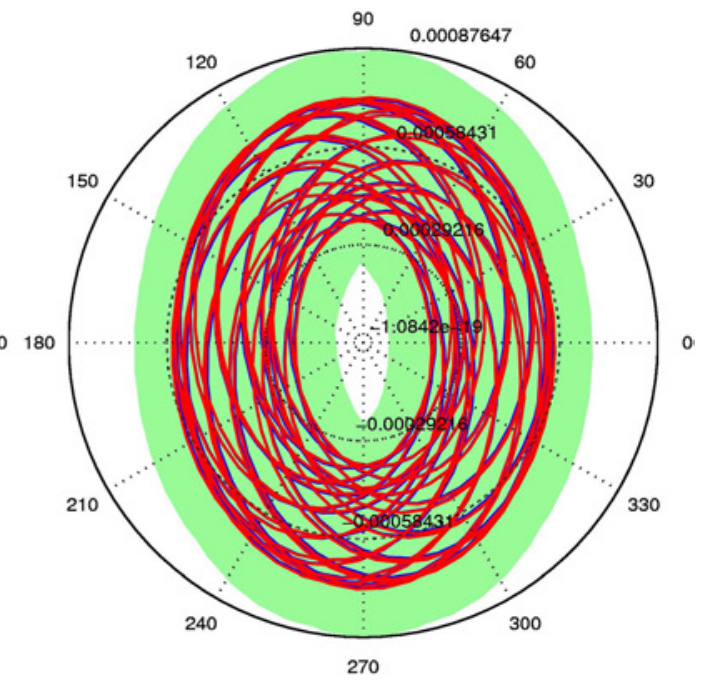

(b)

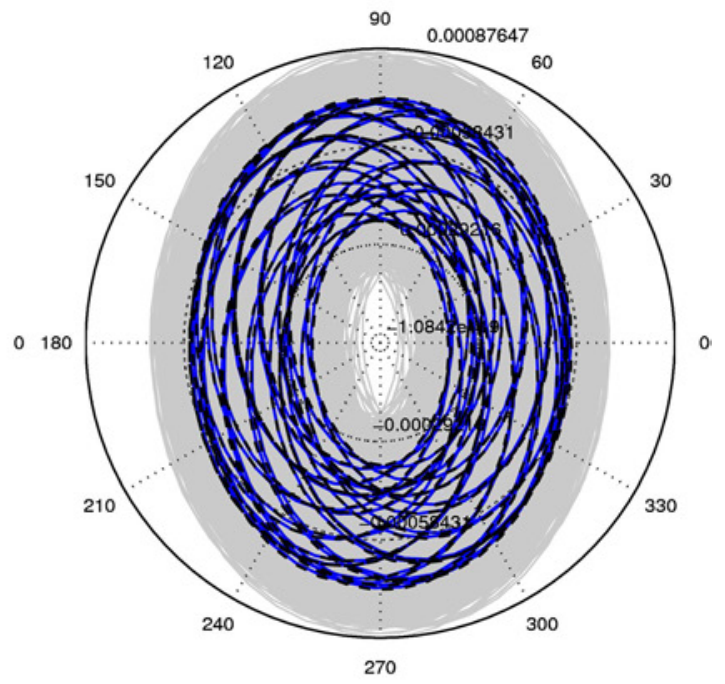

(d)

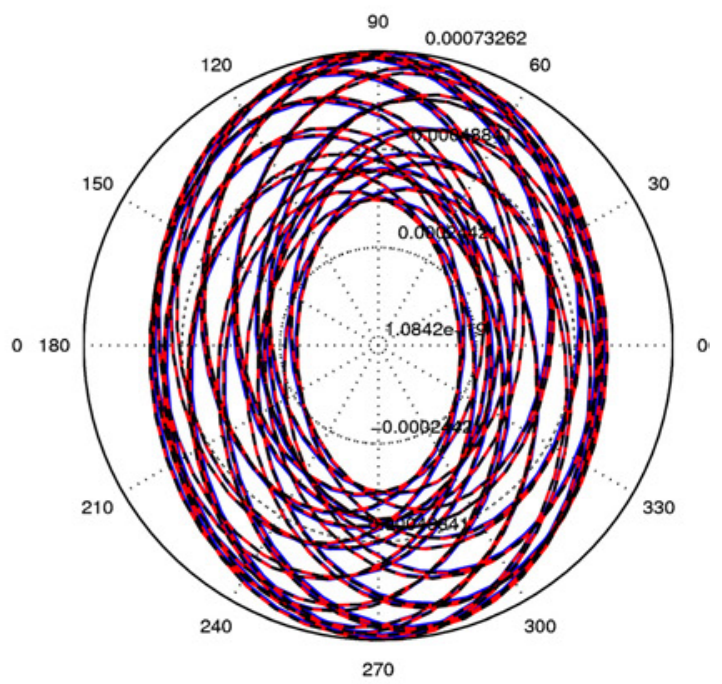

(f)

Fig. 11 Case 1: (a), (c), and (e) orbits at $f_{r}=12.5 \mathrm{~Hz}$ and $f_{b}=100 \mathrm{~Hz}$; $(b)$, (d), and $(f)$ orbits at $f_{r}=64 \mathrm{~Hz}$ and $f_{b}=100 \mathrm{~Hz}$; envelope PCE (a),(b) (green); MC samples (c),(d) (gray lines); deterministic response (all) (blue lines), mean PCE (a), (b), (e), ( $f$ (red lines), mean MCS (c), (d), (e), and ( $f$ ) (dashed black lines) 


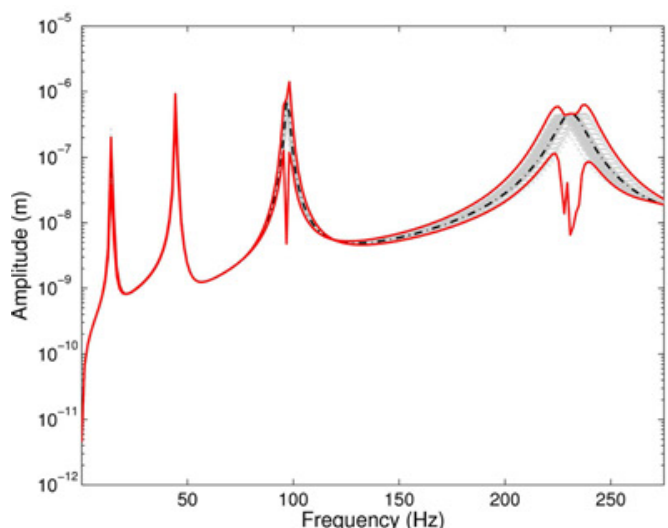

(a)

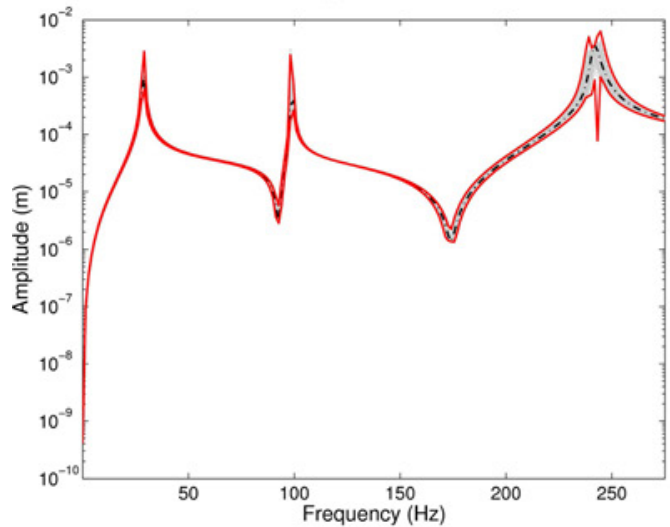

(c)

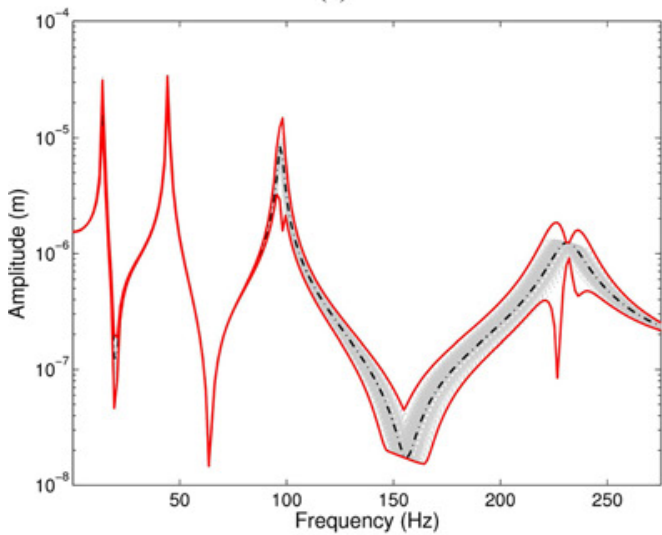

(e)

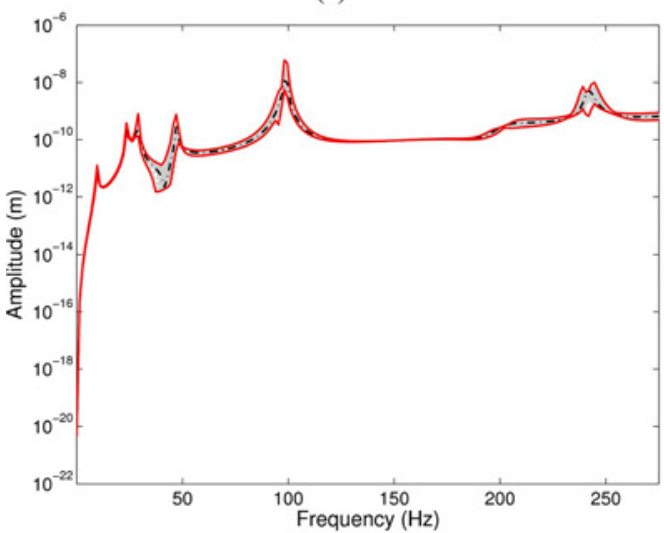

(g)

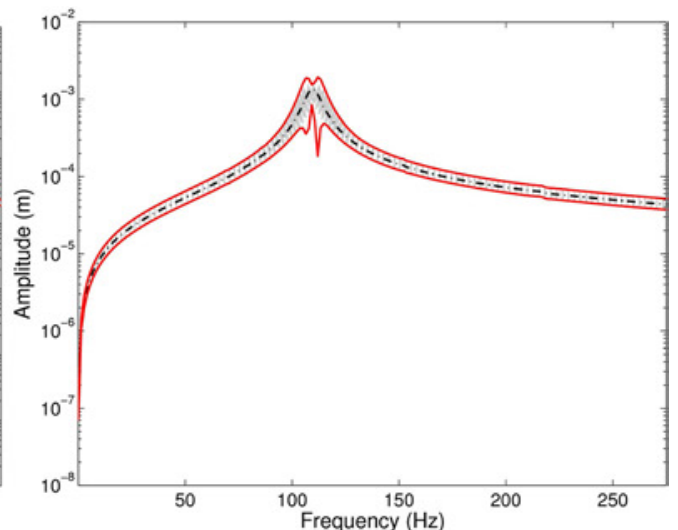

(b)

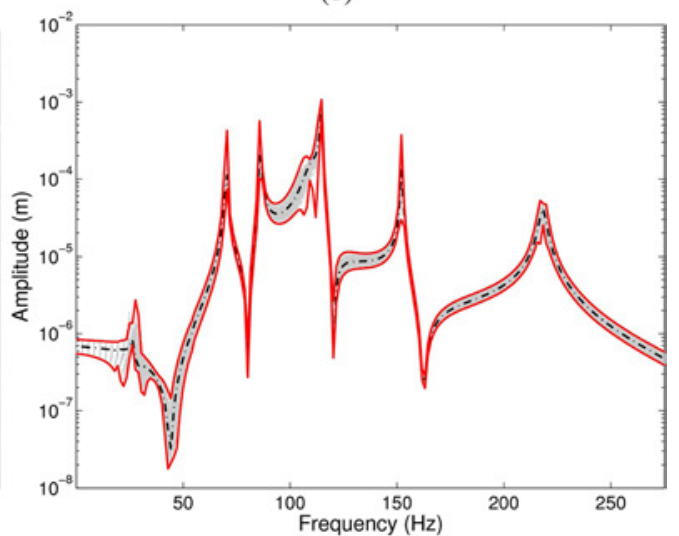

(d)

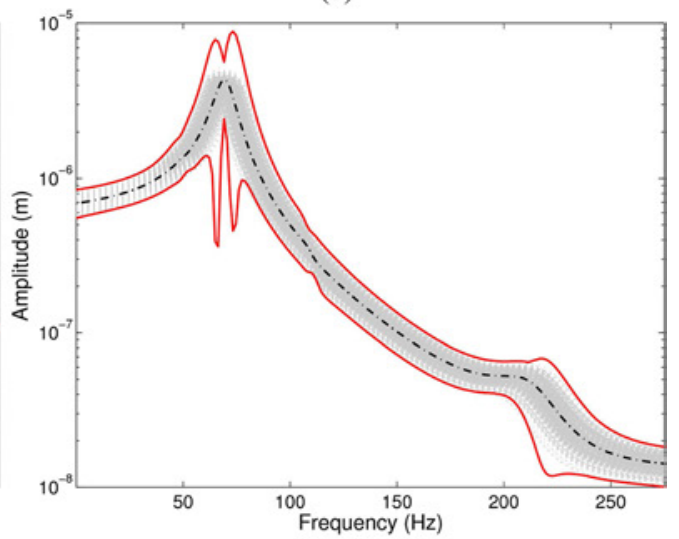

(f)

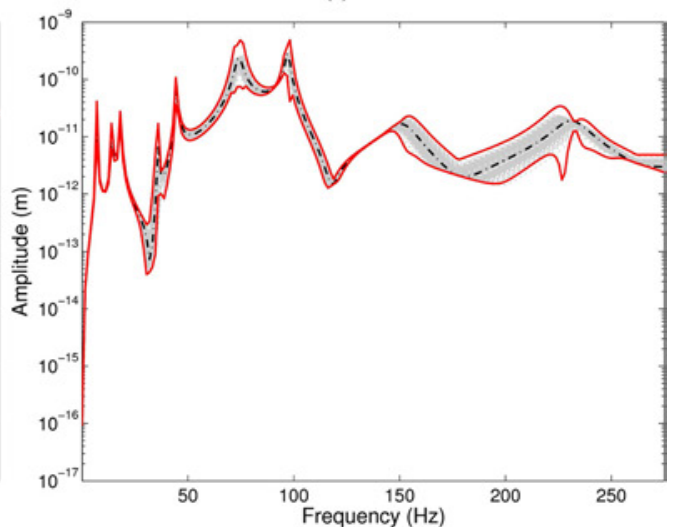

(h)

Fig. 12 Frequency Response Function (case 2 and $f_{b}=100 \mathrm{~Hz}$ ); envelope PCE (red solid lines), MCS (dotted gray lines), deterministic response (dashed-dotted black lines); order $\left[k_{1}, k_{2}\right](a)[0,0],(b)$ $[0,1],(c)[1,0],(d)[2,-1],(e)[2,0],(f)[2,1],(g)[3,0]$, and $(h)[4,0]$ 


$$
\begin{aligned}
& \tilde{\mathbf{G}}=\sum_{j=0}^{P} \tilde{\tilde{\mathbf{G}}}_{j} \Psi_{j}, \quad \tilde{\mathbf{M}}_{0}=\sum_{j=0}^{P} \tilde{\tilde{\mathbf{M}}}_{0_{j}} \Psi_{j}, \quad \tilde{\mathbf{M}}_{c}=\sum_{j=0}^{P} \tilde{\tilde{\mathbf{M}}}_{c_{j}} \Psi_{j}, \\
& \tilde{\mathbf{M}}_{s}=\sum_{j=0}^{P} \tilde{\tilde{\mathbf{M}}}_{s_{j}} \Psi_{j}
\end{aligned}
$$

where $\tilde{\tilde{\mathbf{G}}}_{j}, \tilde{\tilde{\mathbf{M}}}_{0_{j}}, \tilde{\tilde{\mathbf{M}}}_{c_{j}}$ and $\tilde{\tilde{\mathbf{M}}}_{s_{j}}$ are known after the same previous identification.

Finally, the $\tilde{\mathbf{L}}$ operator of the system to be solved is equal to

$$
\tilde{\mathbf{L}}(\tau)=\sum_{i=0}^{P} \mathbf{L}_{i} \Psi_{i}
$$

where components $\mathbf{L}_{i}$ can be obtained using Eqs. (18) with expressions detailed in Appendix A, also valid in the stochastic domain.

3.4 Uncertainties on the Forces. In this section, we focus on the influence of the random character of the forces (applied on the supports excitation and unbalance force) on the response of the system. Firstly, we consider the magnitude of the force as stochastic, so we have

$$
\tilde{U}_{0}(\tau)=\overline{U_{0}}\left(1+\delta_{U_{0}} \xi_{3}\right)
$$

Consequently, the excitation force can be deduced from Eq. (44) into Eq. (8) by

$$
\tilde{\mathbf{f}}^{b}=k_{y y} \overline{U_{0}}\left(1+\delta_{U_{0}} \xi_{3}\right)\left[\begin{array}{llll}
0 & \cos \left(\omega_{b} t\right) & 0 & 0
\end{array}\right]^{T}
$$

Secondly, the unbalance force can be often subject to uncertainties through its mass. We have

$$
\tilde{m}_{e}(\tau)=\bar{m}_{e}\left(1+\delta_{m} \xi_{4}\right)
$$

Then, substituting Eq. (46) into Eq. (2), we obtain

$$
\tilde{\mathbf{f}}^{d}=\bar{m}_{e} d_{e} \omega_{r}^{2}\left(1+\delta_{m} \xi_{4}\right)\left[\begin{array}{llll}
\cos \left(\omega_{r} t\right) & \sin \left(\omega_{r} t\right) & 0 & 0
\end{array}\right]^{T}
$$

Finally, the total force $\tilde{\mathbf{f}}$ applied on the system is the summation of $\tilde{\mathbf{f}}^{b}$ and $\tilde{\mathbf{f}}^{d}$ given by Eqs. (45) and (47). Its expression then depends on random variables $\xi_{3}$ and $\xi_{4}$ which belong to the polynomial chaos basis of random variables set $\left\{\xi_{i}\right\}, i=1$ to 4 . Then, we can expand the global force $\tilde{\mathbf{f}}$ as

$$
\tilde{\mathbf{f}}=\sum_{j=0}^{P} \tilde{\tilde{\mathbf{f}}}_{j} \Psi_{j}
$$

where the coefficients $\tilde{\tilde{\mathbf{f}}}_{j}$ are found by identification between Eq. (48) and the summation of Eqs. (45) and (47).

\section{Results}

In this section, the relevance of the proposed method (MultiHarmonic Balance Method with the Polynomial Chaos Expansion) will be illustrated through two sets of parameters for which uncertainty values are given in Table 3 . It is noted that the order of chaos is set at 2 for all results presented. This choice is guided by the fact that preliminary studies, not presented here, for various orders of chaos led to the conclusion that an order 2 is sufficient in our cases. To validate our approach, we compare the results of the Multi-Harmonic Balance Method with the Polynomial Chaos Expansion, with results obtained by Monte Carlo Simulations that can be costly in computation time.

All values of the physical parameters are given in Table 4 .

4.1 Quasi-Periodic Responses. At first, we propose to focus on the overall quasi-periodic response of the rotor system for case 1. Results are presented at node 6 in the $x$ direction. Figure 4 shows the evolutions of the mean and the variation of the amplitude versus the rotational speed of the rotor system and the excitation frequency of the rotor base, which constitute the two excitations present in the rotor system. It appears that the dynamic response can be very complex: the amplitude level increases of the mean and variation depend on both the excitation frequency of the support and the unbalance excitation (i.e. rotation speed of the system). We can note an important variation when the rotor system is passing through the forward and backward modes for which the fundamental frequencies change depending on the rotational speed of the rotor. It is interesting to note that the appearances and increases of mean and variation when the rotor is near backward modes are directly related to the presence of the rotor asymmetry (See Ref. [11], pages 67-87). In general, the strong trends in the mean and the variation correspond to the response of order 1 (i.e. $\left[k_{1}, k_{2}\right]=[0,1]$ where $k_{1}$ and $k_{2}$ respectively denote order of the term related to the pulsation of the rotor and the excitation pulse of the rotor base). In addition to these variations, small changes are also observable: they correspond to multi-frequency responses of higher orders (i.e. $\left[k_{1}, k_{2}\right]=[n, m]$ with $\left.n+m \geq 2\right)$ and come from the combined contributions of the rotor asymmetry and all the excitations due to unbalance and the base support.

To better visualize these results and to propose a more detailed analysis of the contribution of each order $\left[k_{1}, k_{2}\right]$, we investigate the global quasi-periodic response and the response of each order $\left[k_{1}, k_{2}\right]$ for a fixed excitation frequency of the rotor base. The values of excitation frequencies of the base used in the following results are $100 \mathrm{~Hz}$ and $200 \mathrm{~Hz}$. In this part of the study, it is noteworthy that only harmonics of order $\left[k_{1}, k_{2}\right]$ that are not equal to zero are discussed. The results are presented in the form of the Frequency Response Functions for each harmonic component $\left[k_{1}, k_{2}\right]$ with the upper and lower envelopes obtained via the

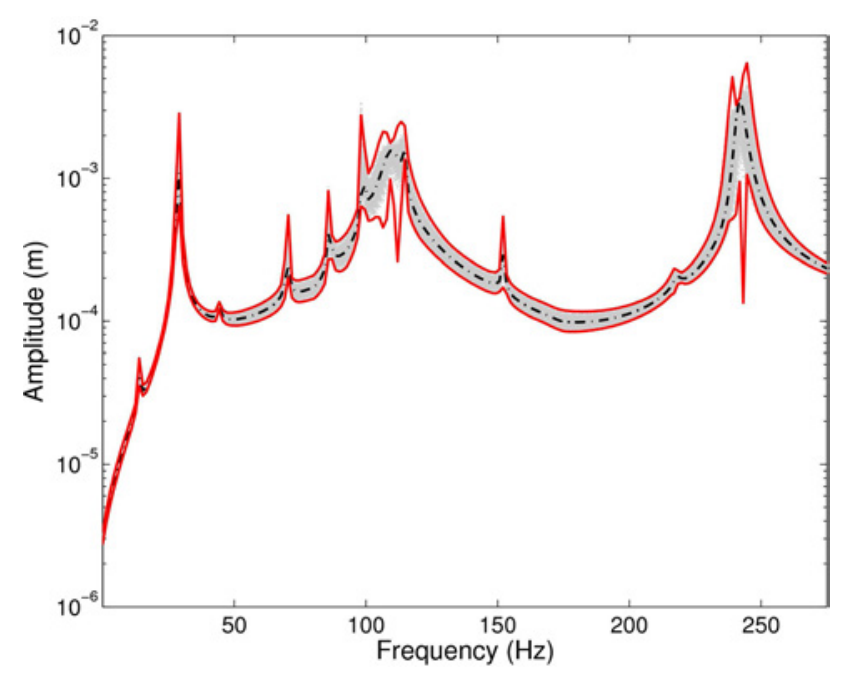

Fig. 13 Frequency Response Function (case 2 and $f_{b}=100$ $\mathrm{Hz}$ ); global quasi-periodic response, envelope PCE (red solid lines), MCS (dotted gray lines), deterministic response (dasheddotted black line) 


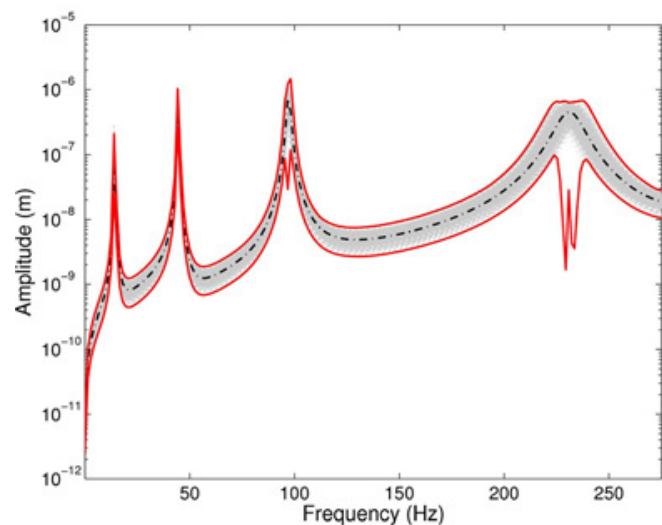

(a)

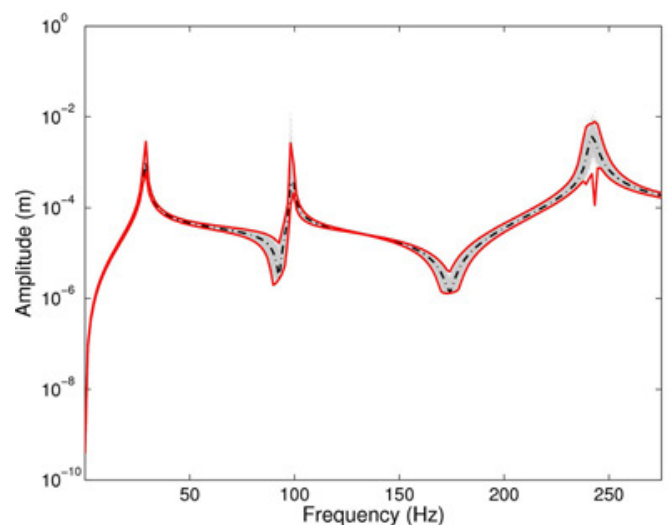

(c)

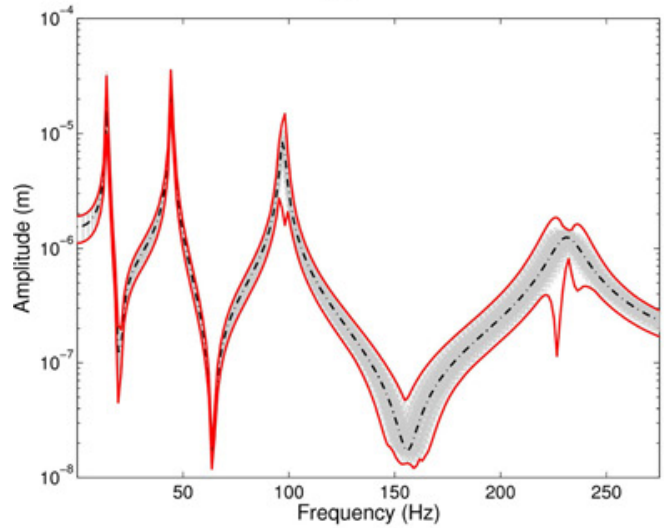

(e)

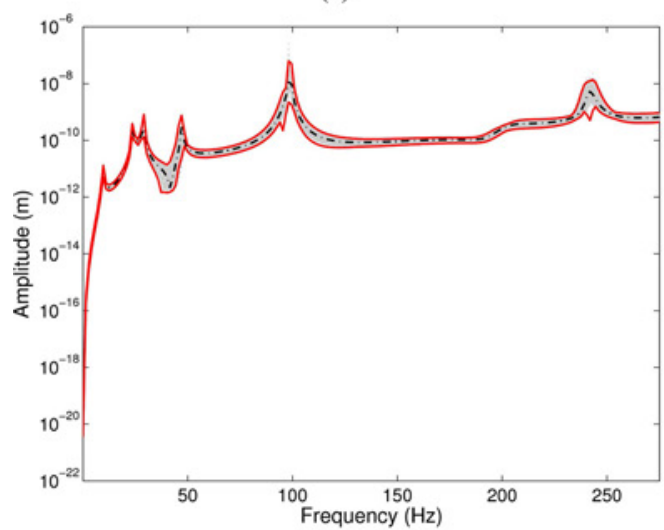

(g)

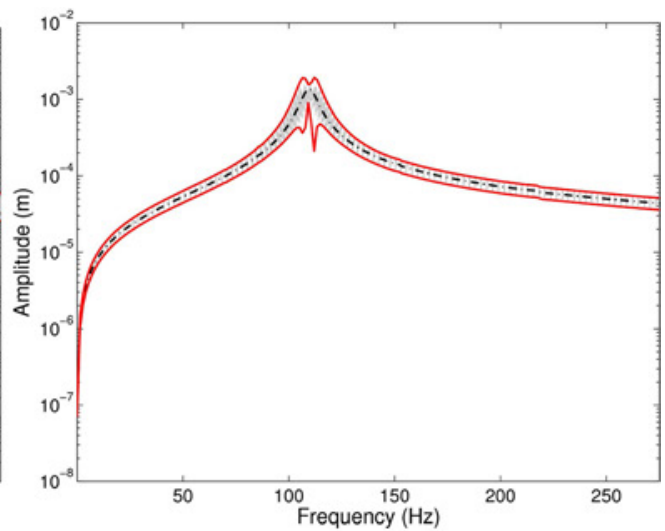

(b)

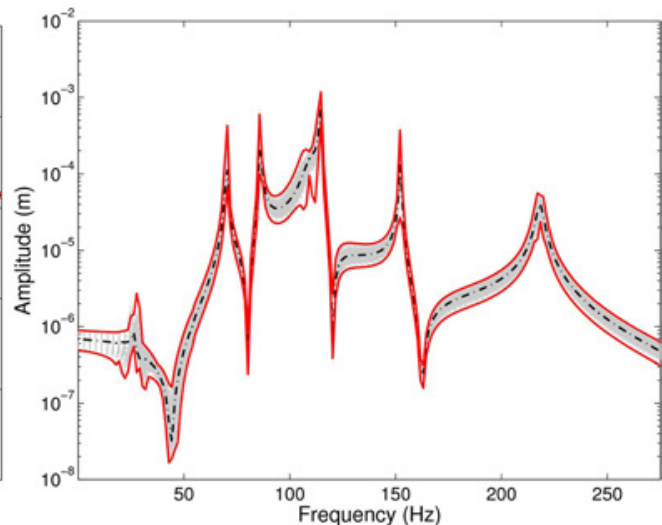

(d)

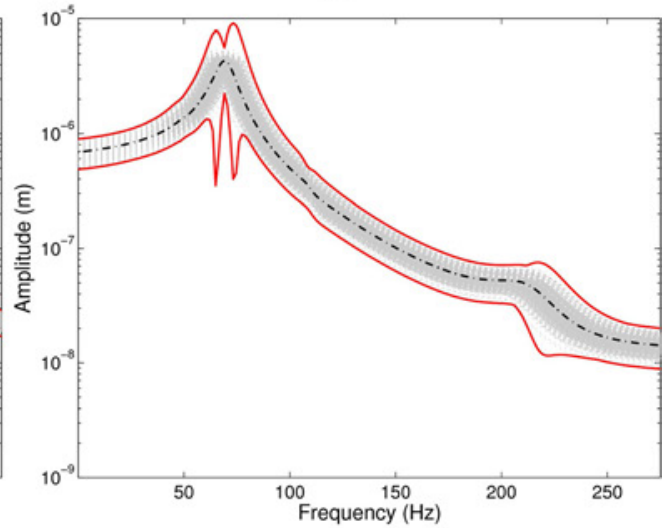

(f)

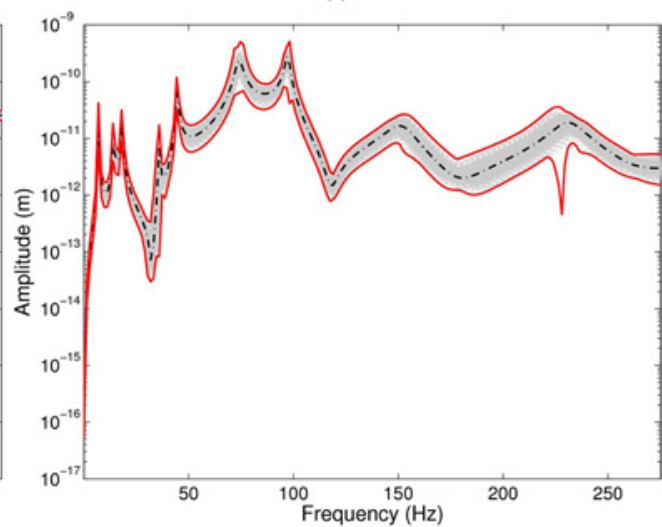

(h)

Fig. 14 Frequency Response Function (case 2 and $f_{b}=200 \mathrm{~Hz}$ ); envelope PCE (red solid lines), MCS (dotted gray lines), deterministic response (dashed-dotted black lines); order $\left[k_{1}, k_{2}\right](a)[0,0]$, (b) $[0,1],(c)[1,0],(d)[2,-1],(e)[2,0],(f)[2,1],(g)[3,0]$, and $(h)[4,0]$ 


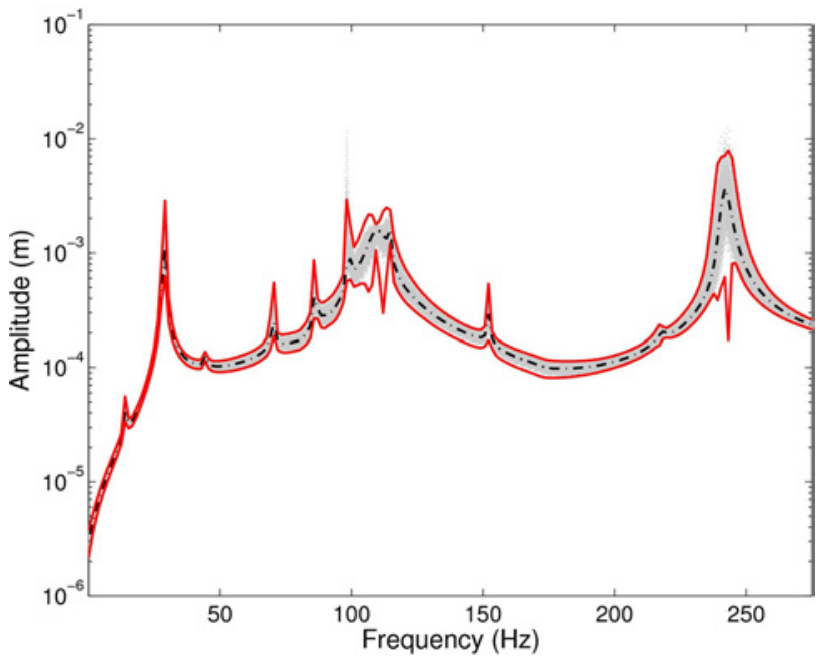

Fig. 15 Frequency Response Function (case 2 and $f_{b}=\mathbf{2 0 0}$ $\mathrm{Hz}$ ); global quasi-periodic response, envelope PCE (red solid lines), MCS (dotted gray lines), deterministic response (dasheddotted black line)

Polynomial Chaos approach, and the form of orbits (for a given rotation speed).

4.2 Rotor Base Excitation at $\mathbf{1 0 0} \mathbf{H z}$. For an excitation frequency of the rotor base equal to $100 \mathrm{~Hz}$, Figs. 5-6 present the results obtained by the approach of the Multi-Dimensional Harmonic Balance Method with a Polynomial Chaos Expansion, and the Monte Carlo Simulations for the first case defined in Table 3. All the quasi-periodic responses obtained by MCS are plotted by gray lines. For the results via the PCE, we show the envelopes that define the values of the minimum and maximum amplitudes. These results are also studied in terms of the evolutions of mean and variation of the amplitudes, as shown in Figs. 7-8.

First, we note that changes in mean and variation of the vibrational responses obtained by the approach PCE are similar to results from the Monte Carlo simulations, for both the global quasi-periodic response and each order $\left[k_{1}, k_{2}\right]$ (see Fig. 7). These comparisons allow us to validate the methodology proposed in this study. Similarly, it clearly appears that the envelopes of the minimum and maximum amplitudes shown in Figs. 5 contain all the responses obtained through the Monte Carlo procedure.

As previously indicated, the predominant vibration amplitudes correspond to the responses of order 1 (see Figs. 5(b) and 5(c)). We also note the significant evolutions of orders $[0,1],[2,1]$, and $[2,-1]$. The presence of these orders is due to the combination of the asymmetry of the rotor with excitations of the rotor support and the unbalance. Also, due to the addition of an uncertainty on the Young's modulus of the shaft, dispersion of amplitudes are visible for all orders, as shown in Fig. 5. However, we note that the dispersion is slightly larger for orders $[0,1],[2,1]$, and $[2,-1]$. This fact can be easily explained by the presence of uncertainty on the excitation amplitude of the rotor support that causes predominant changes for the response of multi-frequency orders with $k_{2} \neq 0$. Finally, Fig. 6 indicates that the quasi-periodic response is very complex and involves non-negligible contributions for orders $\left[k_{1}, k_{2}\right]$ with $k_{1} \neq 0$ and $k_{2} \neq 0$.

Figures $9-10$ present the results obtained by the PCE and MCS approaches for the second set of random parameters (case 2 in Table 3). The remarks previously stated remain valid: this allows us to conclude on the robustness of the pro- posed methodology. The Multi-Dimensional Harmonic Balance method combined with the Polynomial Chaos Expansion provides an effective estimation of the upper and lower envelopes of the quasi-periodic response and overall contributions of harmonics.

In addition, we notice a slight enlargement of the operating area (i.e. increase in the area between the minimum and maximum values of envelopes) for all the nonlinear global responses and the associated contributions of orders. This fact can be explained by the addition of uncertainties on the unbalance and asymmetry of the rotor shaft. Of course, this trend is more pronounced for the responses of orders $\left[k_{1}, k_{2}\right]=[1,0]$ (see Fig. $9(c)$ ) and $\left[k_{1}, k_{2}\right]=[0,2]$ (see Fig. $9(e)$ ) due to the fact that these combinations of orders are directly related to the unbalance excitation and the contribution of the rotor asymmetry, respectively.

Figures 11(a), 11(c), and 11(e) show the orbits obtained for a rotating speed of $12.5 \mathrm{~Hz}$ and a rotor excited at its support at a frequency of $100 \mathrm{~Hz}$. Figures $11(b), 11(d)$, and $11(f)$ give the results when the rotor operates at a rotating speed of $64 \mathrm{~Hz}$. The results (i.e. envelope and mean response) obtained by the MultiDimensional Harmonic Balance Method with the Polynomial Chaos Expansion are illustrated in Figs. 11(a) and 11(b). Responses from the Monte Carlo Simulations are presented in Figs. 11(c) and $11(d)$. Finally, the deterministic response and the mean for both the two various methods are plotted Figs. 11(e) and $11(f)$. We note that the results via the proposed methodology and the Monte Carlo Simulations are similar for both envelopes of the orbits and the mean value of the quasi-periodic dynamical response. These results are quite remarkable because of the complexity of the orbits and envelopes generated (see Figs. 11(a) and $11(c)$ for an example). This demonstrates once again the effectiveness and robustness of the proposed approach based on the combination of the Multi-Dimensional Harmonic Balance Method and the Polynomial Chaos Expansion. So, we can conclude that the proposed methodology can produce results relevant not only for the Frequency Response Functions of the global quasi-periodic responses and each combination of orders (or evolutions of mean and variance, as well as upper and lower envelopes) but also for a description and temporal evolution of orbits.

4.3 Rotor Base Excitation at $200 \mathrm{~Hz}$. Finally, we illustrate the results for an excitation of the rotor base frequency equal to 200 Hz. Figures 12-15 show the Frequency Response Functions for each combination of orders and the global quasi-periodic solution for cases 1 and 2 (Table 3)), respectively. As discussed above, the effectiveness of the novel methodology is demonstrated by the similarity of all the results with those obtained from a conventional Monte Carlo method. In addition, we can notice that the results are close to the previous results for an excitation frequency of $100 \mathrm{~Hz}$. However, different levels for orders $[0,1],[2,1]$, and $[2,-1]$ are observable. This can be explained simply by the fact that the value of the excitation frequency of the rotor support is different. So responses undergo a change of orders.

Finally, evolutions of the orbit shape for various rotational speed and frequency excitation of the base are proposed. Figures 16(a), 16(c), and 16(e) show the orbits obtained for a rotational speed of $12.5 \mathrm{~Hz}$ and a rotor excited at its support at $200 \mathrm{~Hz}$. Then, Figs. $16(b), 16(d)$, and $16(f)$ show the results for a rotational speed of $64 \mathrm{~Hz}$. Considering the previous results presented in Fig. 11 (when the rotor was excited at its support at $100 \mathrm{~Hz}$ ), we can note an evolution of the orbit shape. However, the conclusions about the efficiency of the proposed approach stated above are still valid. Indeed, we note again that the envelopes and the mean of the evolution of orbits obtained by the proposed method are similar to those obtained by the Monte Carlo Simulations. 


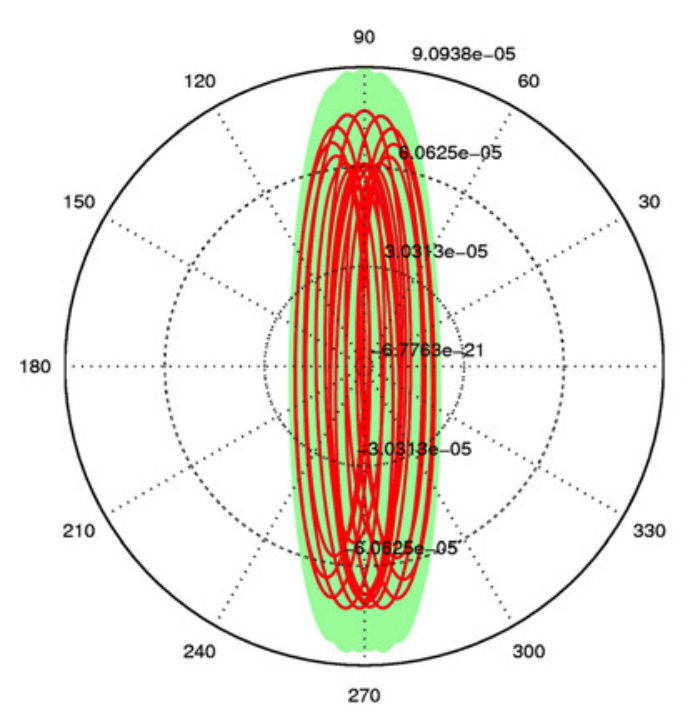

(a)

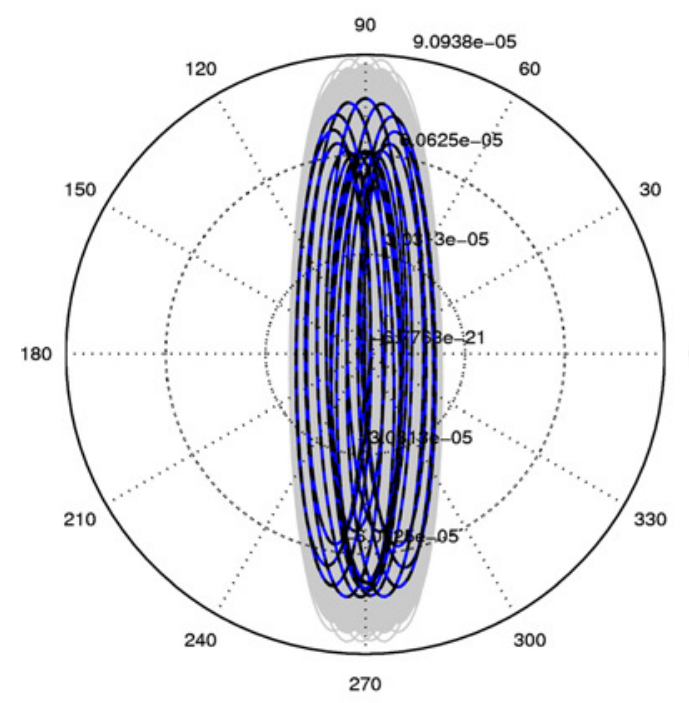

(c)

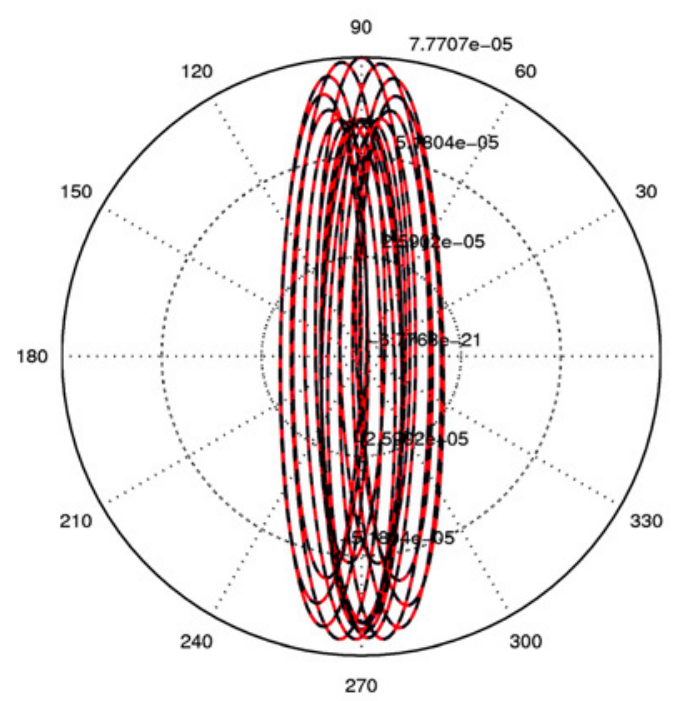

(e)

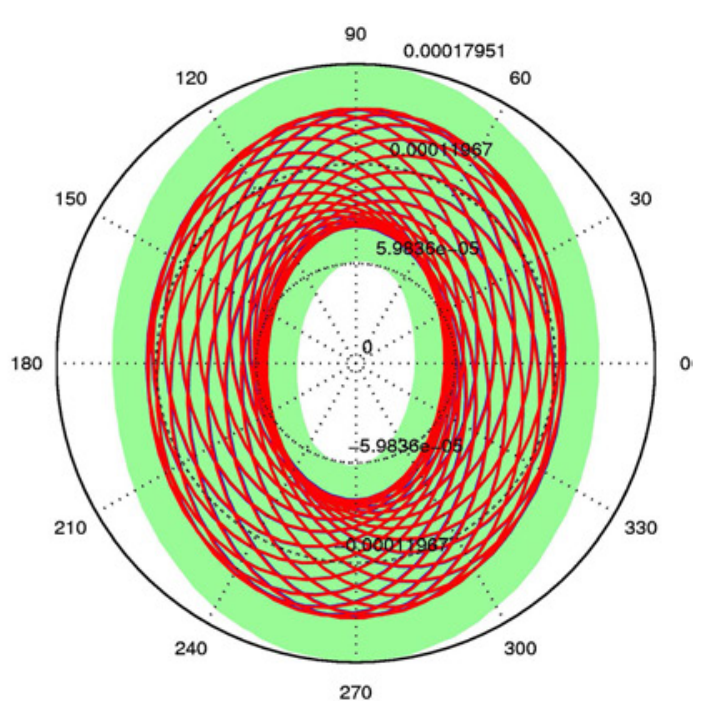

(b)

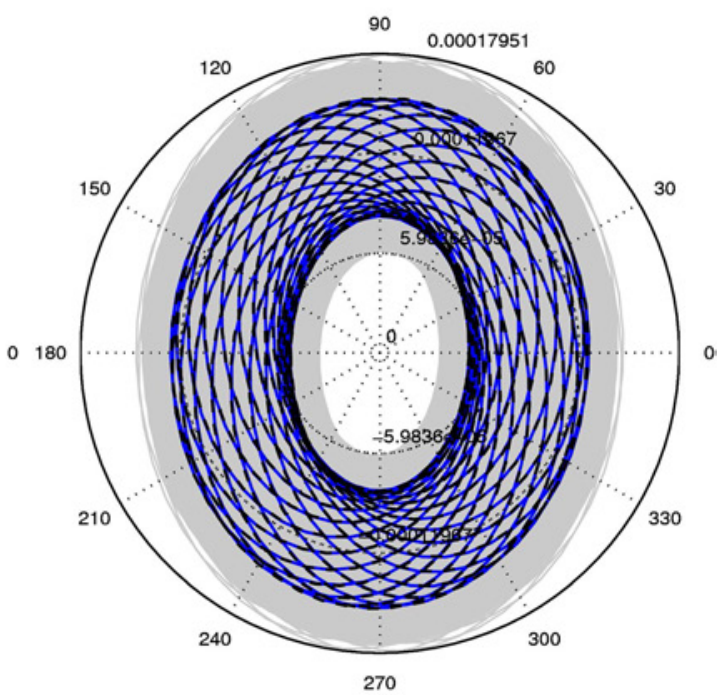

(d)

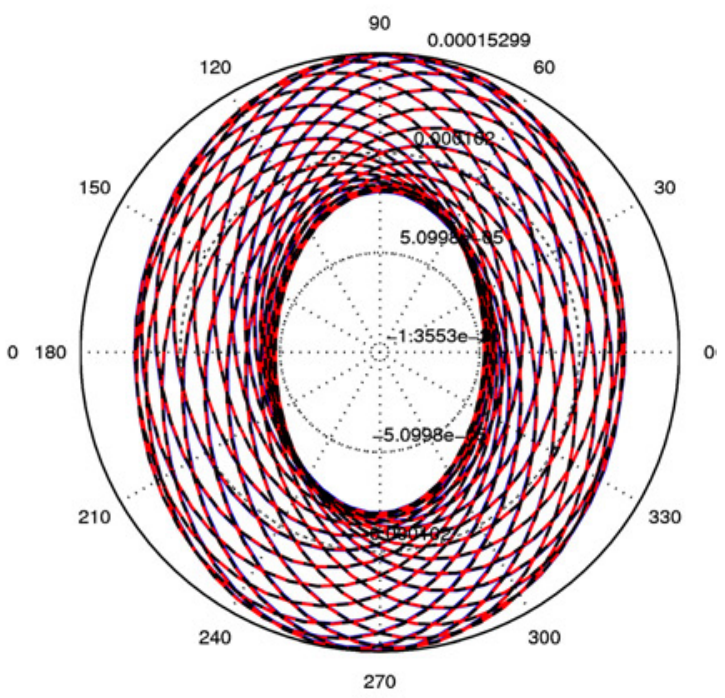

(f)

Fig. 16 Case 1: (a), (c), and (e) orbits at $f_{r}=12.5 \mathrm{~Hz}$ and $f_{b}=200 \mathrm{~Hz} ;(b),(d)$, and $(f)$ orbits at $f_{r}=64 \mathrm{~Hz}$ and $f_{b}=100 \mathrm{~Hz}$; envelope PCE (a),(b) (green); MC samples (c), (d) (gray lines); deterministic response (all) (blue lines), mean PCE (a), $(b),(e)$, and $(f)$ (red lines), mean MCS $(c),(d),(e)$, and ( $f$ ) (dashed black lines) 


\section{Conclusion}

This study describes a novel approach to obtain quasi-periodic responses of mechanical systems with uncertainties. It is based on the Multi-Dimensional Harmonic Balance Method and the Polynomial Chaos Expansion. This methodology is applied to a rotor system in order to estimate the [m,n]-FRF (i.e. Frequency Response Functions of orders [m,n] associated with a twodimensional time domain) and orbits when the rotor is subjected to two excitations (unbalance and excitation at its support) with several material and geometrical random parameters. Effectiveness of the proposed method was demonstrated by comparing the results with those estimated by Monte Carlo Simulations.

Finally, these results illustrate that the presence of uncertainties can clearly affect the quasi-periodic dynamic responses of rotor systems, confirming the need to take into account the variability and randomness of physical parameters in a design stage of a mechanical system.

\section{Acknowledgment}

The first author gratefully acknowledges the French Education Ministry which supports this research. The authors gratefully acknowledge the financial support of the French National Research Agency through the Young Researcher program ANR-07-JCJC-0059-01-CSD2.

\section{Nomenclature}

$$
\begin{aligned}
\mathbf{x} & =\text { displacement vector } \\
\dot{\mathbf{x}} & =\text { velocity vector } \\
\ddot{\mathbf{x}} & =\text { acceleration vector } \\
\mathbf{f} & =\text { forces vector } \\
\mathbf{M} & =\text { mass matrix } \\
\mathbf{G} & =\text { gyroscopic matrix } \\
\mathbf{K} & =\text { stiffness matrix } \\
\mathbf{C} & =\text { damping matrix } \\
\omega_{b} & =\text { support pulsation } \\
\omega_{r} & =\text { rotor pulsation } \\
x^{T} & =\text { transposition operator } \\
N_{h} & =\text { order of the Fourier series } \\
\mathbf{A}_{\mathbf{k}} & =\text { Fourier coefficients of the cosines function for the order } \mathbf{k} \\
\mathbf{B}_{\mathbf{k}} & =\text { Fourier coefficients of the sinus function for the order } \mathbf{k} \\
\Psi_{j} & =\text { the } j^{t h} \text { Hermite polynomial } \\
\underline{\xi}(\tau) & =\text { vector of Gaussian random variables }
\end{aligned}
$$

\section{Appendix: Matrices $I_{1}, I_{2}$, and $I_{3}$}

In this section, the following notations for $\mathbf{X}$ and $\mathbf{x}$ are used

$$
\mathbf{X}=\left[\begin{array}{lll}
\ldots \mathbf{A}_{\mathrm{k}} & \mathbf{B}_{\mathrm{k}} \ldots
\end{array}\right]^{T}=\left[\begin{array}{llll}
\mathbf{A}_{[0,0]} & \mathbf{A}_{[0,1]} & \mathbf{B}_{[0,1]} \ldots \mathbf{A}_{\left[N_{h}, 0\right]} & \mathbf{B}_{\left[N_{h}, 0\right]}
\end{array}\right]^{T}
$$

and

$$
\mathbf{x}(t)=\sum_{\mathbf{k} \in \mathbb{Z}^{p}}\left(\mathbf{A}_{\mathbf{k}} \cos (\mathbf{k} \cdot \omega t)+\mathbf{B}_{\mathbf{k}} \sin (\mathbf{k} \cdot \omega t)\right)
$$

with $\boldsymbol{\omega}=\left[\omega_{r}, \omega_{b}\right]$ and $\mathbf{k}=\left[k_{1}, k_{2}\right]$.

A.1 Matrix $\mathbf{I}_{1}$. The constant terms in the equation of motion given in Eq. (9) are given by expressions $\mathbf{M}_{\mathbf{0}} \ddot{\mathbf{x}}+\left(\mathbf{C}+\omega_{r} \mathbf{G}\right) \dot{\mathbf{x}}+\mathbf{K}_{\mathbf{0}} \mathbf{x}$ and $\mathbf{M}_{\mathbf{0}} \ddot{\mathbf{x}}+\mathbf{D} \dot{\mathbf{x}}+\mathbf{K}_{\mathbf{0}} \mathbf{x}$. This expression can be given as a linear system $\mathbf{I}_{1} \mathbf{X}$ by using the approximated multi-Fourier series for $\ddot{\mathbf{x}}, \dot{\mathbf{x}}$ and $\mathbf{x}$ :

$$
\mathbf{I}_{\mathbf{1}} \mathbf{X}=\left[\begin{array}{cccccc}
\mathbf{K}_{\mathbf{0}} & \mathbf{0} & \mathbf{0} & \mathbf{0} & \mathbf{0} & \mathbf{0} \\
\mathbf{0} & \Lambda_{[0,1]} & \mathbf{0} & \mathbf{0} & \mathbf{0} & \mathbf{0} \\
\mathbf{0} & \mathbf{0} & \ddots & \mathbf{0} & \mathbf{0} & \mathbf{0} \\
\mathbf{0} & \mathbf{0} & \mathbf{0} & \Lambda_{\left[k_{1}, k_{2}\right]} & \mathbf{0} & \mathbf{0} \\
\mathbf{0} & \mathbf{0} & \mathbf{0} & \mathbf{0} & \ddots & \mathbf{0} \\
\mathbf{0} & \mathbf{0} & \mathbf{0} & \mathbf{0} & \mathbf{0} & \Lambda_{\left[N_{h}, 0\right]}
\end{array}\right]\left[\begin{array}{c}
\mathbf{A}_{[0,0]} \\
\mathbf{A}_{[0,1]} \\
\mathbf{B}_{[0,1]} \\
\vdots \\
\mathbf{A}_{\left[k_{1}, k_{2}\right]} \\
\mathbf{B}_{\left[k_{1}, k_{2}\right]} \\
\vdots \\
\mathbf{A}_{\left[N_{h}, 0\right]} \\
\mathbf{B}_{\left[N_{h}, 0\right]}
\end{array}\right]
$$

with $\quad \Lambda_{\left[k_{1}, k_{2}\right]}=\left[\begin{array}{cc}\mathrm{K}_{0}-(\mathrm{k} \cdot \omega)^{2} \mathrm{M}_{0} & \mathrm{k} \cdot \omega \mathrm{D} \\ -\mathrm{k} \cdot \omega \mathrm{D} & \mathrm{K}_{0}-(\mathrm{k} \cdot \omega)^{2} \mathbf{M}_{0}\end{array}\right]$

where $\mathbf{k}=\left[k_{1}, k_{2}\right]$ and $\omega=\left[\omega_{r}, \omega_{b}\right]$

A.2 Matrix $\mathbf{I}_{\mathbf{2}}$. The parametric terms defined by $\left[\mathbf{K}_{\mathbf{c}} \cos \left(2 \omega_{r} t\right)+\mathbf{K}_{\mathbf{s}} \sin \left(2 \omega_{r} t\right)\right]_{\mathbf{k} \in \mathbb{Z}^{p}}\left(\mathbf{A}_{\mathbf{k}} \cos (\mathbf{k} \cdot \omega t)+\mathbf{B}_{\mathbf{k}} \sin (\mathbf{k} \cdot \omega t)\right)$ can be approximated, after calculations, in a multi-Fourier basis as a linear system $\mathbf{I}_{2} \mathbf{X}$ defined by the following expression for $k_{1}>2$

$$
\mathbf{I}_{2} \mathbf{X}=\frac{1}{2}\left[\begin{array}{ccccc}
\mathbf{0} & \mathbf{0} & \Gamma & \mathbf{0} & \mathbf{0} \\
\mathbf{0} & \mathbf{0} & \mathbf{0} & \ddots & \mathbf{0} \\
\ddots & \mathbf{0} & \mathbf{0} & \mathbf{0} & \ddots \\
\mathbf{0} & \ddots & \mathbf{0} & \mathbf{0} & \mathbf{0} \\
\mathbf{0} & \mathbf{0} & \Pi & \mathbf{0} & \mathbf{0}
\end{array}\right]\left[\begin{array}{c}
\mathbf{A}_{\left[k_{1}-2, k_{2}\right]} \\
\mathbf{B}_{\left[k_{1}-2, k_{2}\right]} \\
\mathbf{A}_{\left[k_{1}-1, k_{2}\right]} \\
\mathbf{B}_{\left[k_{1}-1, k_{2}\right]} \\
\mathbf{A}_{\left[k_{1}, k_{2}\right]} \\
\mathbf{B}_{\left[k_{1}, k_{2}\right]} \\
\mathbf{A}_{\left[k_{1}+1, k_{2}\right]} \\
\mathbf{B}_{\left[k_{1}+1, k_{2}\right]} \\
\mathbf{A}_{\left[k_{1}+2, k_{2}\right]} \\
\mathbf{B}_{\left[k_{1}+2, k_{2}\right]}
\end{array}\right]
$$

with $\Gamma=\left[\begin{array}{cc}\mathbf{K}_{c} & \mathbf{K}_{s} \\ -\mathbf{K}_{s} & \mathbf{K}_{c}\end{array}\right]$ and $\Pi=\left[\begin{array}{cc}\mathbf{K}_{c} & -\mathbf{K}_{s} \\ \mathbf{K}_{s} & \mathbf{K}_{c}\end{array}\right]$.

A.3 Matrix $\mathbf{I}_{3}$. The parametric terms defined by $\left(\mathbf{M}_{\mathbf{c}} \cos \left(2 \omega_{r} t\right)+\mathbf{M}_{\mathbf{s}} \sin \left(2 \omega_{r} t\right)\right) \ddot{\mathbf{x}}$ can be approximated, after calculations, in a multi-Fourier basis as a linear system $\mathbf{I}_{3} \mathbf{X}$ given by

$$
I_{3} \mathbf{X}=\frac{1}{2}\left[\begin{array}{ccccc}
\mathbf{0} & \mathbf{0} & \mathbf{H}_{\left[k_{1}, k_{2}\right]} & \mathbf{0} & \mathbf{0} \\
\mathbf{0} & \mathbf{0} & \mathbf{0} & \ddots & \mathbf{0} \\
\ddots & \mathbf{0} & \mathbf{0} & \mathbf{0} & \ddots \\
\mathbf{0} & \ddots & \mathbf{0} & \mathbf{0} & \mathbf{0} \\
\mathbf{0} & \mathbf{0} & \mathbf{K}_{\left[k_{1}, k_{2}\right]} & \mathbf{0} & \mathbf{0}
\end{array}\right]\left[\begin{array}{c}
\mathbf{A}_{\left[k_{1}-2, k_{2}\right]} \\
\mathbf{B}_{\left[k_{1}-2, k_{2}\right]} \\
\mathbf{A}_{\left[k_{1}-1, k_{2}\right]} \\
\mathbf{B}_{\left[k_{1}-1, k_{2}\right]} \\
\mathbf{A}_{\left[k_{1}, k_{2}\right]} \\
\mathbf{B}_{\left[k_{1}, k_{2}\right]} \\
\mathbf{A}_{\left[k_{1}+1, k_{2}\right]} \\
\mathbf{B}_{\left[k_{1}+1, k_{2}\right]} \\
\mathbf{A}_{\left[k_{1}+2, k_{2}\right]} \\
\mathbf{B}_{\left[k_{1}+2, k_{2}\right]}
\end{array}\right]
$$

$$
\begin{aligned}
& \text { with } \mathbf{H}_{\left[k_{1}, k_{2}\right]}=-\left(k_{1} \omega_{r}+k_{2} \omega_{b}\right)^{2}\left[\begin{array}{cc}
\mathbf{M}_{c} & \mathbf{M}_{s} \\
-\mathbf{M}_{s} & \mathbf{M}_{c}
\end{array}\right] \text { and } \mathbf{K}_{\left[k_{1}, k_{2}\right]} \\
& =-\left(k_{1} \omega_{r}+k_{2} \omega_{b}\right)^{2}\left[\begin{array}{cc}
\mathbf{M}_{c} & -\mathbf{M}_{s} \\
\mathbf{M}_{s} & \mathbf{M}_{c}
\end{array}\right] .
\end{aligned}
$$

\section{References}

[1] Didier, J., Sinou, J.-J., and Faverjon, B., 2008, "Study of the Non-Linear Dynamic Response of a Rotor System with Faults and Uncertainties," J. Sound Vib., 331(3), pp. 671-703.

[2] Guskov, M., Sinou, J.-J., and Thouverez, F., 2008, "Multi-Dimensional Harmonic Balance Applied to Rotor Dynamics," Mech. Res. Commun., 35, pp. 537-545.

[3] Benaroya, H., and Rehak, M., 1988, "Finite Element Methods in Probabilistic Structural Analysis: A Selective Review," Appl. Mech. Rev., 41(5), pp. 201-213.

[4] Yamazaki, F., Shinozuka, M., and Dasgupta, G., 1988, "Neumann Expansion for Stochastic Finite Element Analysis," ASCE J. Eng. Mech., 114(8), pp. 1335-1354.

[5] Ghanem, R., and Spanos, P., 1991, Stochastic Finite Elements: A Spectral Approach, Springer-Verlag, Berlin. 
[6] Duchemin, M., Berlioz, A., and Ferraris, G., 2006, "Dynamic Behavior and Stability of a Rotor under Base Excitation," J. Vib. Acoust. 128(5), p. 576-586.

[7] Lalanne, M., and Ferraris, G., 1990, Rotordynamics-Prediction in Engineering, John Wiley \& Sons, New York.

[8] Oncescu, F., Lakis, A. A., and Ostiguy, G., 2001, "Investigation of the Stability and Steady State Response of Asymmetric Rotors, using Finite Element Formulation,” J. Sound Vib., 245(2), pp. 303-328.
[9] Coudeyras, N., Nacivet, S., and Sinou, J.-J., 2009, "Periodic and Quasi-Periodic Solutions for Multi-Instabilities Involved in Brake Squeal," J. Sound Vib. 328(4-5), pp. 520-540

[10] Loève, M., 1977, Probability Theory, 4th ed., Springer-Verlag, Berlin.

[11] Yamamoto, T., and Ishida, Y., 2001, Linear and Nonlinear Rotordynamics. A Modern Treatment With Applications, John Wiley \& Sons, New York. 